\title{
Automorphic forms and rational homology 3-spheres
}

\author{
FRANK CALEGARI \\ NATHAN M DUNFIELD
}

\begin{abstract}
We investigate a question of Cooper adjacent to the Virtual Haken Conjecture. Assuming certain conjectures in number theory, we show that there exist hyperbolic rational homology 3-spheres with arbitrarily large injectivity radius. These examples come from a tower of abelian covers of an explicit arithmetic 3-manifold. The conjectures we must assume are the Generalized Riemann Hypothesis and a mild strengthening of results of Taylor et al on part of the Langlands Program for $\mathrm{GL}_{2}$ of an imaginary quadratic field.
\end{abstract}

The proof of this theorem involves ruling out the existence of an irreducible two dimensional Galois representation $\rho$ of $\operatorname{Gal}(\overline{\mathbb{Q}} / \mathbb{Q}(\sqrt{-2}))$ satisfying certain prescribed ramification conditions. In contrast to similar questions of this form, $\rho$ is allowed to have arbitrary ramification at some prime $\pi$ of $\mathbb{Z}[\sqrt{-2}]$.

In the next paper in this volume, Boston and Ellenberg apply pro- $p$ techniques to our examples and show that our result is true unconditionally. Here, we give additional examples where their techniques apply, including some non-arithmetic examples.

Finally, we investigate the congruence covers of twist-knot orbifolds. Our experimental evidence suggests that these topologically similar orbifolds have rather different behavior depending on whether or not they are arithmetic. In particular, the congruence covers of the non-arithmetic orbifolds have a paucity of homology.

57M27, 11F80, 11F75

\section{Introduction}

Here we investigate questions adjacent to the Virtual Haken Conjecture, which concerns (immersed) surfaces in 3-manifolds. Let $M$ be a closed 3-manifold, that is, one that is compact and has no boundary. An embedded orientable surface $S \neq S^{2}$ in $M$ is incompressible if $\pi_{1}(S) \rightarrow \pi_{1}(M)$ is injective. The manifold $M$ is called Haken if it is irreducible and contains an incompressible surface. A Haken 3-manifold necessarily has infinite fundamental group, but there are many such manifolds which are not Haken. One of the most interesting conjectures about 3-manifolds is Waldhausen's Conjecture [46] which posits the following: 


\subsection{Virtual Haken Conjecture Suppose $M$ is an irreducible 3-manifold with infinite} fundamental group. Then $M$ has a finite cover which is Haken.

Here, the term "virtual" refers to being allowed to pass to finite covers. For such questions, we can always take $M$ to be orientable, and will do so from now on. Assuming the Geometrization Conjecture, a proof of which has been announced by Perelman [32; 33], the unknown (and generic!) case of Conjecture 1.1 is when $M$ is hyperbolic, that is, has a Riemannian metric of constant curvature -1 . Equivalently, $M=\mathbb{H}^{3} / \Gamma$ where $\mathbb{H}^{3}$ is hyperbolic 3-space, and $\Gamma$ is a torsion-free uniform lattice in $\operatorname{Isom}^{+}\left(\mathbb{H}^{3}\right) \cong \mathrm{PSL}_{2}(\mathbb{C}) \cong \mathrm{PGL}_{2}(\mathbb{C})$.

We focus on a stronger form of Conjecture 1.1, which posits the existence of interesting homology in a finite cover:

1.2 Virtual Positive Betti Number Conjecture Let $M$ be a closed hyperbolic 3manifold. Then $M$ has a finite cover $N$ where the Betti number

$$
\beta_{1}(N)=\operatorname{dim} H^{1}(N ; \mathbb{Q})>0 .
$$

The connection to the original conjecture is that $\beta_{1}(N)>0$ implies by Poincare duality that $H_{2}(N ; \mathbb{Z}) \neq 0$, and any non-trivial element of the latter group can be represented by an incompressible surface. There are now many classes of examples for which Conjecture 1.2 is known to hold, but there seems to be no general approach. In the case that $\Gamma=\pi_{1}(M)$ is an arithmetic lattice, Conjecture 1.2 can be naturally related to automorphic forms. However, even in the arithmetic setting, Conjecture 1.2 is known only in special cases, eg when the field of definition has a subfield of index 2 (see Millson [30], Labesse-Schwermer [24], Clozel [7], Lubotzky [26], and Rajan [34]).

In Kirby's problem list [23, Problem 3.58], Cooper formulated the following question, which we devote this paper to studying. The injectivity radius of $M$, denoted $\operatorname{injrad}(M)$, is the radius of the largest ball that can be embedded around every point in $M$; equivalently, it is half the length of the shortest closed geodesic in $M$.

1.3 Question (Cooper) Does there exist a constant $K$ such that if $M$ is a closed hyperbolic 3-manifold with injrad $(M)>K$, then $\beta_{1}(M)>0$ ?

A yes answer to this question would imply Conjecture 1.2, because any hyperbolic 3manifold has a congruence cover with injectivity radius bigger than a fixed $K$. However, the general expectation was that the answer to this question is no; Cooper's motivation in formulating it was to illustrate the depth of our ignorance about Conjecture 1.2. The point of this paper is to show that the answer to Question 1.3 is indeed no, assuming 
certain conjectures in number theory. In particular, we need to assume the Generalized Riemann Hypothesis (GRH) and a mild strengthening of results of Taylor et al on part of the Langlands Program (Conjecture 3.2).

A rational homology sphere is a closed orientable 3-manifold with $\beta_{1}=0$. The topological side of our main result is:

1.4 Theorem Assume the GRH and Conjecture 3.2. Then there exists an explicit tower of covers

$$
N_{0} \leftarrow N_{1} \leftarrow N_{2} \leftarrow N_{3} \leftarrow \cdots
$$

so that each $N_{n}$ is a hyperbolic rational homology sphere, and $\operatorname{inj} \operatorname{rad}\left(N_{n}\right) \rightarrow \infty$ as $n \rightarrow \infty$. Each cover $N_{n} \leftarrow N_{n+1}$ is a regular cover with covering group either $\mathbb{Z} / 3 \mathbb{Z}$ or $\mathbb{Z} / 3 \mathbb{Z} \oplus \mathbb{Z} / 3 \mathbb{Z}$. Moreover, the composite cover $N_{0} \leftarrow N_{n}$ is regular.

In the language of lattices, we have a nested sequence of lattices $\Gamma_{n}=\pi_{1}\left(N_{n}\right)$ in $\mathrm{PSL}_{2}(\mathbb{C})$ such that $\bigcap_{n=1}^{\infty} \Gamma_{n}=1$ and $H^{1}\left(\Gamma_{n} ; \mathbb{R}\right)=0$ for each $n$ (equivalently, the abelianization of each $\Gamma_{n}$ is finite). Examples of infinite towers of covers of hyperbolic rational homology spheres have been previously constructed by Baker, Boileau, and Wang [1], but these lack the crucial requirement on the injectivity radius.

Despite Theorem 1.4, the $N_{n}$ are easily seen to satisfy Conjecture 1.2. Amusingly, it turns out that all of the $N_{n}$ are in fact Haken (see Section 2.10). Thus they do not give a no answer to

1.5 Question Does there exist a constant $K$ such that if $M$ is a closed hyperbolic 3-manifold with injrad $(M)>K$, then $M$ is Haken?

As with Cooper's original question, we suspect the answer must be no, but see no way of showing this.

The construction of the examples in Theorem 1.4 is arithmetic in nature. A precise statement is

1.6 Theorem Let $D$ be the (unique) quaternion algebra over $K=\mathbb{Q}(\sqrt{-2})$ ramified at $\pi$ and $\bar{\pi}$, where $3=\pi \bar{\pi}$. Let $B$ be a maximal order of $D$. Let $\mathfrak{m}$ be a maximal bi-ideal of $B$ trivial away from $\pi$. Finally, let $B_{n}$ be the complex embedding of $B^{\times} \cap\left(1+\mathfrak{m}^{n}\right)$ into $\mathrm{PGL}_{2}(\mathbb{C})$, and let $M_{n}=\mathbb{H}^{3} / B_{n}$. Then assuming the Langlands conjecture for $\mathrm{GL}_{2}\left(\mathbb{A}_{K}\right)$ (Conjecture 3.2) and the $G R H$, then $H^{1}\left(M_{n} ; \mathbb{Q}\right)=0$ for all $n$. 
The examples $N_{n}$ of Theorem 1.4 are the above $M_{n}$ with the first few dropped for technical reasons; see Section 2.5 for details. In the next paper in this volume, Boston and Ellenberg apply pro- $p$ techniques to the above $M_{n}$ and show that $H^{1}\left(M_{n} ; \mathbb{Q}\right)=$ 0 unconditionally [2]. We give additional examples where their techniques apply, including some non-arithmetic examples, in Section 6.

It has long been known that the cohomology groups of arithmetic 3-manifolds are related to spaces of automorphic forms. This relationship has been previously exploited: Clozel [7] was able to prove non-vanishing results for certain cohomology groups by using automorphic forms associated to Grössencharakters. In contrast, computations made by Grunewald, Helling, and Mennicke [16], and Cremona [11], working with congruence covers of Bianchi manifolds, suggest the paucity of automorphic forms, and lead to the suspicion that for certain groups, infinitely many congruence covers contain no interesting (non-cuspidal) cohomology. To this point such problems have been unapproachable. The trace formula, so useful in many other situations, here only contributes the equality $0=0$ (Poincare duality identifies the two "interesting" cohomology groups, $H^{1}$ and $H^{2}$ ). In this paper, we present an approach to these questions that succeeds (assuming standard conjectures) in ruling out the existence of automorphic forms associated to particular congruence covers of arbitrarily large degree.

The starting point is the theorem of Taylor et al $[18 ; 40]$ which establishes for the automorphic forms of interest a family of compatible $\operatorname{Gal}(\bar{K} / K)$-representations for some imaginary quadratic field $K$. The nature of these representations is not yet completely understood, and in particular we must assume a slight strengthening of their results. The main idea is that one can rule out the existence of certain automorphic forms by ruling out the existence of the corresponding Galois representations, even allowing the conductor of these representations to become arbitrarily large. This is in contrast to the situation over $\mathbb{Q}$, where applications tend to work in reverse: the proof of Fermat's last theorem [42; 49] uses the non-existence of modular forms of weight two and level $\Gamma_{0}(2)$ to rule out the existence of certain Galois representations! We restrict our representations $\rho$ in several steps. First, choose a prime $p=\pi \bar{\pi}$ that splits in the ring of integers of $K$ and consider automorphic forms whose conductor is highly divisible by $\pi$ but strictly controlled at $\bar{\pi}$; let $\rho$ be the associated $\lambda$-adic representation for some $\lambda \mid p$. Second, using an idea of Tate [39] we prove in a specific case that $\bar{\rho}$ must be reducible. For this step we require the Generalized Riemann Hypothesis. The key is now to use the local behavior of $\rho$ at $\bar{\pi}$ as a fulcrum to prove that $\rho$ is reducible, which is enough to force a contradiction. This last idea in a different guise can be seen in the work of Fontaine [14] (further developed by Schoof and others, see for example Brumer-Kramer [3], Calegari [4] and Schoof [37]). Finally, our application to rational 
homology spheres comes from switching between non-compact covers of Bianchi manifolds and compact arithmetic quotients of $\mathbb{\boxplus}^{3}$ by using the Jacquet-Langlands correspondence. We learnt of this idea from Alan Reid.

Finally, we investigate the congruence covers of twist-knot orbifolds, with the goal being to gather experimental evidence about how common it is for congruence covers to have $\beta_{1}>0$. For instance, one would like to know if it is plausible to attack Conjecture 1.2 solely by examining covers of this kind. (For comparison, complex hyperbolic manifolds are also expected to satisfy Conjecture 1.2. However, there are infinitely many incommensurable arithmetic complex hyperbolic manifolds all of whose congruence covers have $\beta_{1}=0$; see Rapoport-Zink [35], Rogawski [36] and Clozel [8].) Our examination of a family of topologically similar orbifolds found strikingly different behavior depending on whether or not the orbifold was arithmetic. In particular, the congruence covers of the non-arithmetic orbifolds have a paucity of homology. Indeed, it seems plausible that our sample includes a non-arithmetic hyperbolic orbifold where only finitely many congruence covers of the form $\Gamma_{0}(\mathfrak{p})$ have $\beta_{1}>0$.

\subsection{Outline of contents}

Section 2 gives a detailed description of the orbifolds $M_{n}$ and $N_{n}$ of the main theorems from both topological and arithmetic points of view. Section 2 also derives Theorem 1.4 from Theorem 1.6. Section 3 discusses the connection to automorphic forms, states the needed form of the Langlands conjecture, and gives the reduction of Theorem 1.6 to showing the non-existence of certain Galois representations. Such Galois representations are ruled out in Sections 4-5. An elaboration of the Boston-Ellenberg pro- $p$ approach is given in Section 6, together with a list of examples to which it applies. Finally, Section 7 contains the experimental results on the twist-knot orbifolds.

\subsection{Acknowledgments}

Calegari was partially supported as a 5-year fellow of the American Institute of Mathematics. Dunfield was partially supported by U.S. National Science Foundation grant \#DMS-0405491, as well as a Sloan Fellowship. The authors thank Nigel Boston, Kevin Buzzard, Jordan Ellenberg, Oliver Goodman, Damian Heard, Craig Hodgson, Alex Lubotzky, Hee Oh, Dinakar Ramakrishnan, Alan Reid, and Richard Taylor for helpful conversations and correspondence.

\section{The examples}

In this section, we give a detailed description of the orbifolds $M_{n}$ of Theorem 1.6, and also derive Theorem 1.4 from Theorem 1.6. 


\subsection{The orbifold $M_{0}$}

We start by looking at $M_{0}$, which is constructed arithmetically as follows (for details see Machlachlan-Reid [28], and Vigneras [45]). We start with the field $K=\mathbb{Q}(\sqrt{-2})$. The prime 3 splits over $K$ into two primes $\pi=1-\sqrt{-2}$ and $\bar{\pi}=1+\sqrt{-2}$. Let $D$ be the unique quaternion algebra over $K$ which is ramified at exactly the places $\pi$ and $\bar{\pi}$. Explicitly, $D$ has a standard basis $\{1, i, j, i j\}$ where $i^{2}=-1, j^{2}=-3$, and $i j=-j i$; equivalently, $D$ is said to be given by the Hilbert symbol $\left(\frac{-1,-3}{K}\right)$. Let $B$ be a maximal order in $D$. The order $B$ is unique up to conjugacy, as the number of conjugacy classes of maximal orders divides the restricted class number $h_{\infty}$ of $K$, which here is just the class number $h=1$ (see eg Machlachlan-Reid [28, Section 6.7]). Now let $B^{\times}$be the group of units of $B$. Taking the complex place of $K$ gives us an embedding $D \rightarrow D \otimes_{K} \mathbb{C} \cong M_{2}(\mathbb{C})$ into the algebra of $2 \times 2$ matrices over $\mathbb{C}$. The image of $B^{\times}$lands in $\mathrm{GL}_{2}(\mathbb{C})$, and composing this with the projectivization $\mathrm{GL}_{2}(\mathbb{C}) \rightarrow \mathrm{PGL}_{2} \mathbb{C}$ gives a homomorphism $\rho: B^{\times} \rightarrow \operatorname{PGL}(2, \mathbb{C})$. The target group $\mathrm{PGL}_{2} \mathbb{C}$ can naturally be identified with the group of orientation preserving isometries of hyperbolic 3-space $\mathbb{H}^{3}$. The group $\rho\left(B^{\times}\right)$is a lattice, and the base orbifold for our examples is

$$
M_{0}=\mathbb{M}^{3} / \rho\left(B^{\times}\right) .
$$

Since, by construction, $D$ ramifies at some finite place, the orbifold $M_{0}$ is compact.

2.2 Remark In the topology literature, it is more typical to consider $B^{1}$, the elements of $B$ of reduced norm 1, rather than $B^{\times}$. At the complex place, such elements lie in $\mathrm{SL}_{2}(\mathbb{C}) \leq \mathrm{GL}_{2}(\mathbb{C})$, and also give a lattice $\rho\left(B^{1}\right) \leq \rho\left(B^{\times}\right)$in $\mathrm{PGL}_{2}(\mathbb{C})$. The reduced norm gives a homomorphism from $B^{\times}$to the group of units of $K$, which is just \pm 1 . The group $B^{1}$ is the kernel, and hence $\left[\rho\left(B^{\times}\right): \rho\left(B^{1}\right)\right]=2$. Thus, if we set $M_{0}^{\prime}=\mathbb{H}^{3} / \rho\left(B^{1}\right)$, we that have $M_{0}^{\prime}$ is a 2 -fold cover of $M_{0}$. From the point of view of automorphic forms, it is more natural to work with $M_{0}$ rather than $M_{0}^{\prime}$.

The basic topology of $M_{0}$ is also easy to describe. The underlying topological space for $M_{0}$ is just the 3-sphere $S^{3}$, and the orbifold locus is shown in Figure 1 (for the derivation of this from the arithmetic, see Section 2.9). The hyperbolic volume of $M_{0}$ can be directly calculated from data about the quaternion algebra, see eg MachlachlanReid [28, Theorem 11.1.3]:

$$
\operatorname{Vol}\left(M_{0}\right)=\frac{1}{2} \operatorname{Vol}\left(M_{0}^{\prime}\right)=\frac{8 \sqrt{2}}{\pi^{2}} \zeta_{K}(2)=2.0076820066823962745447297 \ldots
$$

Here is a presentation for $\pi_{1}\left(M_{0}\right)=\rho\left(B^{\times}\right)$:

$$
\left\langle u, v, x, y \mid u^{2}, v^{2}, x^{4}, y^{4}, y x y^{-1} v x^{-1} v, x^{-1} v x v u y^{-1} u y,\left(u y^{-1} u y\right)^{3}\right\rangle
$$




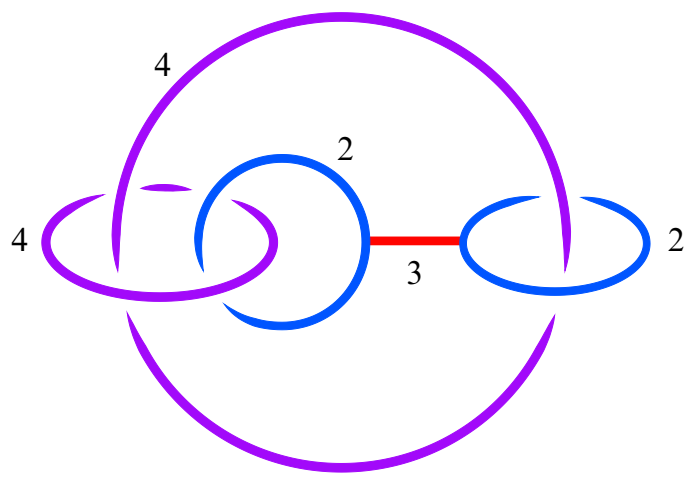

Figure 1: The orbifold $M_{0}=\mathbb{H}^{3} / \rho\left(B^{\times}\right)$has underlying space $S^{3}$ with orbifold locus the above graph. The labels on the edges of the graph correspond to the order of the cyclic group action associated to that part of the singularity.

which is obtained by eliminating excess generators from a Wirtinger presentation derived from Figure 1. Let's now give the canonical representation $\pi_{1}\left(M_{0}\right) \rightarrow \operatorname{PSL}_{2}(\mathbb{C})$ in terms of the quaternion algebra. The maximal order $B$ can be taken to be the $\mathcal{O}_{K}$-span of

$$
\langle 1, i, s=(1 / 2)(i+j), t=(1 / 2)(1+i j)\rangle .
$$

As discussed in Section 2.9, in terms of our presentation for $\pi_{1}\left(M_{0}\right)$ the unit group $B^{\times}$is generated by

$$
\begin{array}{lll}
u \mapsto i, & v \mapsto-2 i+\pi s, \\
x \mapsto \sqrt{-2} \cdot 1+\pi i+\bar{\pi} s-\sqrt{-2} t, & y \mapsto \sqrt{-2} \cdot 1+s-\sqrt{-2} t .
\end{array}
$$

The representation $\pi_{1}\left(M_{0}\right) \rightarrow \mathrm{PSL}_{2}(\mathbb{C})$ is then obtained by taking an explicit embedding of $D$ into $M_{2}(\mathbb{C})$, eg

$$
i \mapsto\left(\begin{array}{cc}
i & 0 \\
0 & -i
\end{array}\right), \quad j \mapsto\left(\begin{array}{cc}
0 & 1 \\
-3 & 0
\end{array}\right) .
$$

\subsection{The orbifolds $M_{n}$}

We turn now to the orbifolds $M_{n}$. By definition $M_{n}$ is the congruence cover of $M_{0}$ of level $\pi^{n}$. Very succinctly, this means that if $\mathfrak{m}$ is the maximal bi-ideal of $B$ trivial away from $\pi$, then $M_{n}=\mathbb{M}^{3} / \rho\left(B^{\times} \cap\left(1+\mathfrak{m}^{n}\right)\right)$. We will now describe these congruence covers in more detail, so that we may derive Theorem 1.4 from Theorem 1.6. It is worth noting that since these are congruence covers for a prime that ramifies in $D$, their structure is rather different than those for a generic prime. 
As usual, $K_{\pi}$ will denote the completion of $K$ with respect to the $\pi$-adic norm. The integers in $K$ will be denoted $\mathcal{O}=\mathbb{Z}[\sqrt{-2}]$, the $\pi$-adic completion of $\mathcal{O}$ is the valuation ring $\mathcal{O}_{\pi}$. As $\mathcal{O}$ has unique factorization, we conflate primes ideals and prime elements, so that $\pi$ is also a uniformizing element in $\mathcal{O}_{\pi}$, with $\pi \mathcal{O}_{\pi}$ the unique maximal ideal. Now consider $D$ at this finite place, which we denote $D_{\pi}=K_{\pi} \otimes_{K} D$. That $D$ ramifies at $\pi$ means precisely that $D_{\pi}$ is the unique division algebra over $K_{\pi} \cong \mathbb{Q}_{3}$. Explicitly, $D_{\pi}$ is the following (for details, see Maclachlan-Reid [28, Section 2.6, Section 6.4] which we follow closely). Let $L$ be the unique unramified quadratic extension of $K_{\pi}$; then $L=K_{\pi}(\sqrt{u})$ for some unit $u \in \mathcal{O}_{\pi}^{\times}$. Explicitly, $D_{\pi}$ is specified by the Hilbert symbol $\left(\frac{u, \pi}{K_{\pi}}\right)$. Even more concretely, one can take

$$
D_{\pi}=\left\{\left(\begin{array}{cc}
a & b \\
\pi b^{\prime} & a^{\prime}
\end{array}\right)\right\} \quad \text { where } a, b \in L
$$

and ' denotes the Galois involution of $L / K$. In this model, $\{1, i, j\}$ correspond to $(a, b)=(1,0),(\sqrt{u}, 0)$, and $(0,1)$ respectively. A concise way of writing an element of $D_{\pi}$ is thus $a+b j$ where $a, b \in L$. The reduced norm $n$ is then $a a^{\prime}-\pi b b^{\prime}$, and the trace $a+a^{\prime}$.

The algebra $D_{\pi}$ has a natural norm $|d|=|n(d)|_{\pi}$ where $|\cdot|_{\pi}$ is the norm on $K_{\pi}$. The valuation ring $B_{\pi}=\left\{d \in D_{\pi}|| d \mid \leq 1\right\}$ is the unique maximal order of $D_{\pi}$. In (2.6), the elements of $B_{\pi}$ are simply those that have $a, b \in \mathcal{O}_{L}$. We have a natural embedding $B \hookrightarrow B_{\pi}$; as $B$ is maximal in $D$, the valuation ring $B_{\pi}$ is equal to $B \otimes_{\mathcal{O}} \mathcal{O}_{\pi}$. The unique maximal bi-ideal of $B_{\pi}$ is just $Q=B_{\pi} j$. The units $B_{\pi}^{\times}$have a natural filtration

$$
B_{\pi}^{\times} \supset 1+Q \supset 1+Q^{2} \supset 1+Q^{3} \supset \cdots
$$

Let $\Gamma_{n}$ be the preimage of $1+Q^{n}$ under $B^{\times} \hookrightarrow B_{\pi}$, and set $M_{n}=\mathbb{M}^{3} / \rho\left(\Gamma_{n}\right)$. By definition, $M_{n}$ is the congruence cover of $M_{0}$ of level $\pi^{n}$. To relate this back to language at the beginning of this subsection, the bi-ideal $\mathfrak{m}$ of $B$ is precisely the preimage of $Q$ under $B \hookrightarrow B_{\pi}$, and so $\Gamma_{n}=B^{\times} \cap\left(1+\mathfrak{m}^{n}\right)$.

We now examine the orbifolds $M_{n}$ more closely, and so derive Theorem 1.4 from Theorem 1.6.

Proof of Theorem 1.4 The manifold $N_{n}$ in the statement of the theorem is simply $M_{n+d}$ for a fixed positive integer $d$. (We need to drop the first few $M_{n}$ as they are genuine orbifolds, not manifolds.) Thus modulo Theorem 1.6, we need to check three things:

(1) The quotient $M_{n}=\mathbb{H}^{3} / \rho\left(\Gamma_{n}\right)$ is a manifold for large $n$; equivalently $\rho\left(\Gamma_{n}\right)$ is torsion free. 
(2) The injectivity radius of $M_{n}$ goes to $\infty$ as $n \rightarrow \infty$.

(3) The covering group of $M_{n} \leftarrow M_{n+1}$ is $\mathbb{Z} / 3 \mathbb{Z}$ for even $n>0$ and $(\mathbb{Z} / 3 \mathbb{Z})^{2}$ for odd $n>0$. Moreover, $M_{0} \leftarrow M_{n}$ is a regular cover for all $n$.

First, let us examine $M_{0} \leftarrow M_{1}$. Consider the residue field of $L$, denoted $\bar{L}=$ $\mathcal{O}_{L} / \pi \mathcal{O}_{L} \cong \mathbb{F}_{9}$. Then

$$
B_{\pi}^{\times} /(1+Q) \cong \bar{L}^{\times} \cong \mathbb{Z} / 8 \mathbb{Z} \quad \text { via } a+b j \mapsto a+\pi \mathcal{O}_{L} .
$$

To determine $\left[B^{\times}: \Gamma_{1}\right]$, we need to understand the image of $B^{\times}$in $B_{\pi}^{\times}$. This image is not dense as all elements of $B^{\times}$have norm in $\mathcal{O}^{\times}=\{ \pm 1\}$, whereas the set of norms elements is $B_{\pi}^{\times}$is infinite. However, if we let $B_{\pi}^{ \pm 1}$ consist of those elements of norm \pm 1 , then strong approximation (see Machlachlan-Reid [28, Theorem 7.7.5]) shows that $B^{\times}$is dense in $B_{\pi}^{ \pm 1}$. It is not hard to see that $B^{ \pm 1} /(1+Q)$ is still all of $\bar{L}^{\times}$; for this and subsequent calculations, the reader may find it convenient to note $D_{\pi} \cong\left(\frac{-1,3}{\mathbb{Q}_{3}}\right)$. Thus it follows that $B^{\times} \rightarrow \mathbb{Z} / 8 \mathbb{Z}$ is surjective. Thus $\left[B^{\times}: \Gamma_{1}\right]=8$; however $\left[\rho\left(B^{\times}\right): \rho\left(\Gamma_{1}\right)\right]=4$ as the kernel of $\rho: B^{\times} \rightarrow \operatorname{PGL}_{2}(\mathbb{C})$ is $\{ \pm 1\}$, which maps non-trivially under $B^{\times} \rightarrow \mathbb{Z} / 8 \mathbb{Z}$. Thus $M_{0} \leftarrow M_{1}$ is a 4 -fold cyclic cover, and $\rho: \Gamma_{n} \rightarrow \mathrm{PGL}_{2} \mathbb{C}$ is injective for all $n>0$. Notice also that the reduced norm map $n: B^{\times} \rightarrow\{ \pm 1\}$ is the same as the composite $B^{\times} \rightarrow \mathbb{Z} / 8 \mathbb{Z} \rightarrow \mathbb{Z} / 2 \mathbb{Z}$; hence $\Gamma_{n}$ lies in $B^{1}$ for $n>0$.

Now look at $M_{n} \leftarrow M_{n+1}$. In this case, we have

$$
1 \rightarrow \Gamma_{n+1} \rightarrow \Gamma_{n} \rightarrow\left(1+Q^{n}\right) /\left(1+Q^{n+1}\right) \cong \bar{L}^{+} \cong(\mathbb{Z} / 3 \mathbb{Z})^{2},
$$

where the interesting identification is given by $1+(a+b j) j^{n} \mapsto a+\pi \mathcal{O}_{L}$. The image of the rightmost map is $B_{\pi}^{1} /\left(1+Q^{n+1}\right)$, which turns out to be $\mathbb{Z} / 3 \mathbb{Z}$ when $n$ is even and $(\mathbb{Z} / 3 \mathbb{Z})^{2}$ when $n$ is odd. Thus $M_{n} \leftarrow M_{n+1}$ is has covering group either $\mathbb{Z} / 3 \mathbb{Z}$ or $(\mathbb{Z} / 3 \mathbb{Z})^{2}$. Also, since $Q^{n}$ is a bi-ideal, it is immediate that $\Gamma_{n}$ is normal in $B^{\times}$. This establishes point (3) above.

Turning now to (1) and (2), consider some $\Gamma_{n}$ for a fixed $n>0$. As noted above, $\Gamma_{n}$ is in $B^{1}$. Hence for $g \in \Gamma_{n}$, the trace of $g$ is the same the trace of $\rho(g)$ in $\operatorname{PSL}_{2}(\mathbb{C})$ (the latter of which is only defined up to sign). Suppose $g$ is non-trivial. If $g$ has finite order, then $\operatorname{tr}(g)$ is a real number in $(-2,2)$. Otherwise $g$ corresponds to a closed geodesic in $M_{n}$. If the length of this geodesic is $l$ and the twist parameter is $\theta \in[0,2 \pi)$, then

$$
\cosh \left(\frac{l+i \theta}{2}\right)= \pm \frac{\operatorname{tr}(g)}{2}
$$


Thus to prove (1) and (2) it suffices to show that

$$
\min \left\{|\operatorname{tr}(g)| \mid g \in \Gamma_{n} \backslash\{1\}\right\} \rightarrow \infty \quad \text { as } n \rightarrow \infty,
$$

where $|\cdot|$ denotes the absolute value on $K$ at the complex place. Fix $g \in \Gamma_{n} \backslash\{1\}$. Now, as $g=1+x j^{n}$, we have $\operatorname{tr}(g)=2+y \pi^{m}$ where $m=\lceil n / 2\rceil$ and $y \in \mathcal{O}$. Hence

$$
|\operatorname{tr}(g)| \geq|y| \cdot|\pi|^{m}-2 \geq 1 \cdot 3^{m / 2}-2 \geq 3^{n / 4}-2,
$$

and thus (2.8) holds, completing the proof of the theorem.

\subsection{Finding a topological description of $M_{0}$}

In this subsection, we outline the procedure used to find the topological description of $M_{0}$ given in Figure 1. Along the way, we find topological descriptions of $M_{0}^{\prime}=\mathbb{H}^{3} / \rho\left(B^{1}\right)$ and $M_{1}$, the latter of which has the interesting consequence that the examples of Theorem 1.4 are Haken (see Section 2.10).

When trying to determine the topology of $M_{0}$, it is important to remember that the structure of a commensurability class of arithmetic 3-manifolds is quite complicated; in particular, it contains infinitely many minimal orbifolds (throughout this subsection, see Maclachlan-Reid [28] for details). We started by simply finding some arithmetic orbifold commensurable to $M_{0}$. A natural place to look is the Hodgson-Weeks census of small volume closed hyperbolic 3-manifolds [47]; however, the smallest manifold commensurable with $M_{0}$ has much too large a volume to appear there. Instead, we started with cusped manifolds in the Callahan-Hildebrand-Weeks census and did orbifold Dehn filling, looking for something with volume a rational multiple of the value given by (2.3). The commensurability class of an arithmetic hyperbolic 3-orbifolds is completely determined by the invariant trace field and quaternion algebra, which may be computed using Goodman's program Snap $[15 ; 10]$. One thus finds that the orbifold $s 594(3,-3)$ is commensurable with $M_{0}$ and $\operatorname{Vol}(s 594(3,-3))=2 \operatorname{Vol}\left(M_{0}\right)$. However, the (non-invariant) trace field of $s 594(3,-3)$ is bigger than $K$, so $s 594(-3,3)$ is not derived from a quaternion algebra; that is, it is not conjugate into $\rho\left(B^{1}\right)$. Passing to a 2 -fold cover gives an orbifold $N$ which lies in $\rho\left(B^{1}\right)$ with index 2 . Using Thistlethwaite's table of links provided with [15], a brute force search finds the Dehn surgery description of $N$ shown in Figure 2.

Since $N$ is derived from a quaternion algebra, and $\operatorname{Vol}(N)=2 \operatorname{Vol}\left(M_{0}^{\prime}\right)$, we know that there is some involution of $N$ which quotients it down to $M_{0}^{\prime}$. Unfortunately, the symmetry group of $N$ is quiet large $\left(\operatorname{Isom}(N)=\mathbb{Z} / 2 \mathbb{Z} \times D_{8}\right)$, and there are some 11 distinct involutions of $N$. In order to find the correct one, we used Snap to give matrices in $\mathrm{PSL}_{2} \mathbb{C}$ inducing each of these automorphisms of $\pi_{1}(N) \leq \mathrm{PSL}_{2} \mathbb{C}$. Adjoining these 


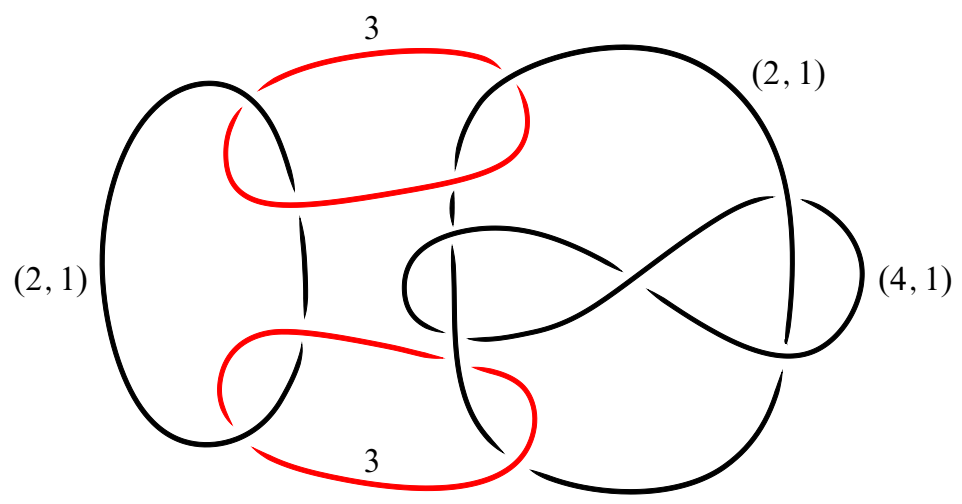

Figure 2: Dehn surgery description of an orbifold $N$, which will be shown to be $M_{1}$. Our framing conventions for Dehn surgery are given by:

to $\pi_{1}(N)$ one at a time, one finds there is a unique one, call it $\tau$, where the trace field remains just $K$. Looking at the action of $\tau$ on short geodesics of $N$ shows that $\tau$ in fact comes from a symmetry of the link in the surgery diagram of Figure 2. The symmetry group of the link is much smaller, namely just $\mathbb{Z} / 2 \mathbb{Z} \oplus \mathbb{Z} / 2 \mathbb{Z}$. One also finds that $\tau$ fixes each component of the orbifold locus of $N$ at exactly two points. This now forces $\tau$ to be the involution of $N$ given in Figure 3. The quotient $N / \tau$ is then $M_{0}^{\prime}=\mathbb{R}^{3} / \rho\left(B^{1}\right)$, which is given in Figure 4.

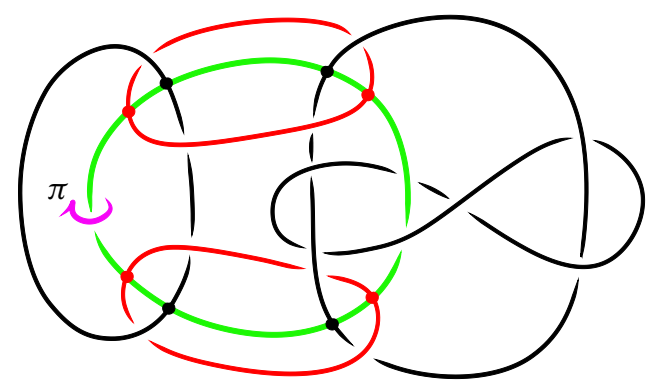

Figure 3: The involution $\tau$ which quotients $N$ down to $M_{0}^{\prime}$. The fixed point set of $\tau$ intersects the link in 8 points.

To find $M_{0}$ itself, one again searches for an additional symmetry $\tau^{\prime}$ to add to $N$ so that $\left\langle\pi_{1}(N), \tau, \tau^{\prime}\right\rangle$ has trace field $\mathbb{Q}(\sqrt{-2}, i)$. One finds that there is essentially only possible choice for $\tau^{\prime}$, and this has order 4 with $\left(\tau^{\prime}\right)^{2}=\tau$. It is not hard to see a symmetry of Figure 2, which quotients down to Figure 1. Since the orbifold in Figure 1 has 4-torsion, is must be the quotient of $N$ under the action of a cyclic group of order 4 . However, there are two distinct $\mathbb{Z} / 4 \mathbb{Z}$ subgroups of $\operatorname{Isom}(N)$ which contain $\tau$, so one must do another check to see that Figure 1 is really $M_{0}^{\prime}$. 


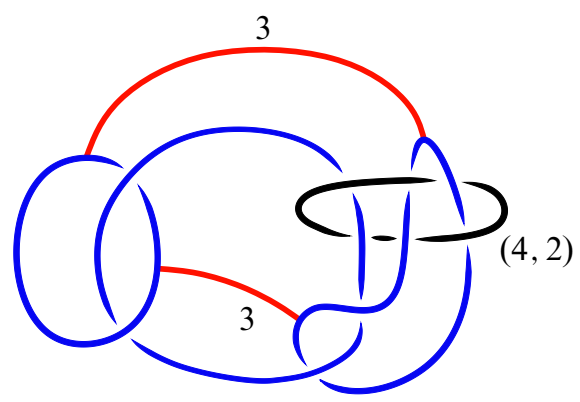

Figure 4: The orbifold $M_{0}^{\prime}=\mathbb{H}^{3} / \rho\left(B^{1}\right)$. The unlabeled part of the singular graph should be labeled 2 .

We started with the presentation (2.4) for the fundamental group of the orbifold give in Figure 1. Eliminating variables, it is possible to solve for a representation $\rho_{0}: \pi_{1} \rightarrow$ $\mathrm{PSL}_{2} \mathbb{C}$ which appears to be the canonical representation. From this, one derives the quaternion algebra picture given at the end of Section 2.1. Thus a posteriori, $\rho_{0}\left(\pi_{1}\right)$ is a discrete group. Using SnapPea [47] to compute a Dirichlet domain shows that $\rho_{0}\left(\pi_{1}\right)$ is cocompact with the correct volume, and hence the claimed generators for $B^{\times}$really do generate it. One then observes that gluing up this Dirichlet domain according to the face pairings gives Figure 1; since residually finite groups are Hopfian it follows that $\rho_{0}$ is faithful. More simply, one can use Heard's new program Orb [19] to see that the canonical representation is as claimed.

To conclude this section, we show that $N$ is none other than $M_{1}$. From the proof of Theorem 1.4, we know that $M_{0} \leftarrow M_{1}$ is cyclic cover of degree 4. Also, the traces of elements of $\Gamma_{1} \leq B^{1}$ are congruent to $2 \bmod \pi$. It follows that $\pi_{1}\left(M_{1}\right)=\rho\left(\Gamma_{1}\right)$ contains no 2-torsion. Now $N$ and the congruence cover of $M_{1}^{\prime}$ corresponding to $\bar{\pi}$ are also 4-fold cyclic covers of $M_{0}$ with no 2-torsion. There are only two candidate homomorphisms $\pi_{1}\left(M_{0}\right) \rightarrow \mathbb{Z} / 4 \mathbb{Z}$ whose kernels contain no 2 -torsion. As $B$ is maximal, strong approximation implies that $M_{1}$ and $M_{1}^{\prime}$ are distinct covers of $M_{0}$, Thus if $N$ is not $M_{1}^{\prime}$, it must be equal to $M_{1}$. A quick check with SnapPea shows that $\pi(N)$ contains elements whose traces are congruent to $0 \bmod \bar{\pi}$, and hence $N \not M_{1}^{\prime}$. (Note: SnapPea only gives elements in $\mathrm{PSL}_{2} \mathbb{C}$, where the trace is defined only up to sign. This does not matter since we are testing whether the trace is 0 in $\mathbb{F}_{3}$.) Thus $N \cong M_{1}$ as desired.

\subsection{Virtual properties of $M_{0}$}

Despite Theorem 1.4, the orbifold $M_{0}$ does satisfy Conjecture 1.2. Indeed, since $K=\mathbb{Q}(\sqrt{-2})$ has a subfield of index two, there are in fact congruence covers with $\beta_{1}>0$ (see Labesse-Schwermer [24] and Lubotzky [26]). One way to see this directly 
is to start from the fact that $M_{0}$ contains an immersed totally geodesic surface. This follows because $D$ is ramified at exactly the two primes sitting over 3 (see MaclachlanReid [28, Theorem 9.5.5]). Concretely, $D$ can also be defined by the Hilbert symbol $(-1,3)$, in addition to $(-1,-3)$; the quaternion algebra defined over $\mathbb{Q}$ with symbol $(-1,3)$ gives the totally geodesic surface. The presence of an immersed totally geodesic surface implies not just Conjecture 1.2, but the stronger statement that $M_{0}$ has a finite cover $N$ where $\pi_{1}(N)$ surjects onto a free group of rank 2 (see Lubotzky [27]). One can also see such a virtually free quotient directly from the topological description of $M_{1}$ given in Figure 2. There, the underlying space of the orbifold

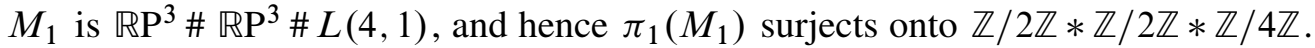
The latter group acts on an infinite 4-valent tree without a global fixed point. For an $M_{n}$ with $n>1$, the covering map $M_{1} \leftarrow M_{n}$ gives an action of $\pi_{1}\left(M_{n}\right)$ on the same tree, again without a global fixed point. Therefore, if $M_{n}$ is a manifold, there is an incompressible surface dual to this action. Thus $M_{n}$ is Haken, and hence all the examples in Theorem 1.4 are Haken.

\section{Modular forms for $\mathrm{GL}_{2} / K$}

Let $K / \mathbb{Q}$ be a number field. We will use $\mathbb{A}_{K}$ to denote the adèles of $K$, and $\mathbb{A}_{K}^{\infty}$ the finite adèles. According to the Langlands philosophy, any regular algebraic cuspidal automorphic representation $\pi$ of $\mathrm{GL}_{2}\left(\mathbb{A}_{K}\right)$ should be attached to a compatible family of $\operatorname{Gal}(\bar{K} / K)$-representations, whose local representations are well behaved and can $a$ priori be determined by the local factors of $\pi$. If $K$ is totally real such representations may be constructed "geometrically" from the Tate modules of certain Shimura varieties (see Carayol [6] and Taylor [41]). We are interested in the case where $K$ is an imaginary quadratic field, and here the corresponding symmetric spaces fail to be algebraic varieties. This causes a great headache in the construction of Galois representations which one expects are always geometric. This problem was solved in the paper [18] by Harris, Soudry and Taylor and the subsequent paper of Taylor [40]. The kernel of the idea is to use automorphic induction to $\mathrm{GSp}_{4} / \mathbb{Q}$ where one has geometry with which to construct Galois representations. The original desired 2-dimensional $\operatorname{Gal}(\bar{K} / K)-$ representation should then be related to these $\mathrm{Gal}(\overline{\mathbb{Q}} / \mathbb{Q})$ representations by Galois induction. The result of these labours is the following theorem [40].

3.1 Theorem (Taylor) Let $K$ be an imaginary quadratic field, and let ${ }^{-}$denote its non-trivial automorphism. Let $\pi$ be a cuspidal automorphic representation of $\mathrm{GL}_{2}\left(\mathbb{A}_{K}\right)$ 
such that $\pi_{\infty}$ has Langlands parameter

$$
W_{\mathbb{C}}=\mathbb{C}^{\times} \rightarrow \mathrm{GL}_{2}(\mathbb{C}) \quad \text { given by } \quad z \mapsto\left(\begin{array}{cc}
z^{1-k} & 0 \\
0 & \bar{z}^{1-k}
\end{array}\right), \quad \text { where } k \in \mathbb{Z}_{\geq 2} .
$$

Let $S$ be a set of places containing those where $K$ ramifies and those where $\pi$ or $\bar{\pi}$ is ramified. For $v \notin S$ let $\left\{\alpha_{v}, \beta_{v}\right\}$ be the Langlands parameters of $\pi_{v}$. Let $F$ be the field generated by $\alpha_{v}+\beta_{v}$ and $\alpha_{v} \beta_{v}$ for $v \notin S$; it is a number field. Assume

(1) the central character $\chi$ of $\pi$ satisfies $\chi=\bar{\chi}$,

(2) the integer $k$ in the Langlands parameter is even.

Then there is an extension $E / F$ such that for each prime $\lambda$ of $F$ there is a continuous irreducible representation

$$
\rho_{\lambda}: \operatorname{Gal}(\bar{K} / K) \rightarrow \mathrm{GL}_{2}\left(E_{\lambda}\right)
$$

such that if $v$ is a prime of $K$ outside $S$ and not dividing the residue characteristic $\ell$ of $\lambda$ then $\rho$ is unramified. Moreover, the characteristic polynomial of $\rho_{\lambda}\left(\mathrm{Frob}_{v}\right)$ is $\left(x-\alpha_{v}\right)\left(x-\beta_{v}\right)$ for a set of places $v$ of density one.

The existence of Galois representations in a setting where the associated symmetric spaces are not algebraic now allows us to study these spaces, which are arithmetic hyperbolic manifolds, using Galois representations. Nevertheless, Theorem 3.1 is not sufficient for our purposes. In order to study more precisely the arithmetic of $\rho$, we need finer control over the behavior of $\rho$ at primes in the set $S$, and the primes dividing the residue characteristic $\ell$. What we need can be described by the following conjectural extension of Theorem 3.1:

3.2 Conjecture (Langlands for $\mathrm{GL}_{2} / K$ ) Let $K$ be an imaginary quadratic field, and let $\pi$ be as in Theorem 3.1, without assuming conditions 1 or 2 . Then the representation $\rho_{\lambda}$ exists as above, and is potentially semistable at $v$ for all $v$. Furthermore, the associated representation of the Weil-Deligne group satisfies the local Langlands correspondence with the associated representation $\pi_{v}$.

One may ask how far Taylor's theorem is from establishing Conjecture 3.2. The condition on the central character seems unavoidable in the arguments of Taylor et al. Beyond this, there are essentially two issues, one minor, one more serious. The first concerns the behavior at a prime $v \nmid \ell$ when $\pi_{v}$ and $\bar{\pi}_{v}$ have different ramification behavior, for example when $\bar{\pi}_{v}$ is ramified and $\pi_{v}$ is not. In this context, Taylor's result does not guarantee (as Conjecture 3.2 does) that $\rho_{\lambda}$ is unramified at $v$. (Note the 
statement of [18, Theorem A] contains a somewhat mischievous misprint: "does not divide $\mathfrak{n} \ell$ " should read "does not divide $N_{K / \mathbb{Q}}(\mathfrak{n}) \ell$ ".) The issue is that the associated form $\tilde{\pi}$ on $\mathrm{GSp}_{4}$ couples $\pi_{v}$ and $\bar{\pi}_{v}$ together, and thus $\widetilde{\pi}_{\ell}$ can be ramified even if $\pi_{v}$ is not. One possible approach would be to exploit the compatibility of local and global Langlands for $\mathrm{GSp}_{4} / \mathbb{Q}$; Taylor has sketched an argument for the first author along these lines. However, such an argument would require a proof of said compatibility for $\mathrm{GSp}_{4} / \mathbb{Q}$, which is not currently known (though not expected to be too difficult, for example it is now known for $\mathrm{GL}_{n}$; see Taylor-Yoshida [43]). A more serious issue arises for $v \mid \ell$, even for $k=2$, the case of interest. Here it is not even known that the $\ell$-adic representations on $\mathrm{GSp}_{4}$ are potentially semistable at $\ell$, since their construction uses congruences to higher weight forms. An analogous issue arises for Hilbert modular forms and was solved also by Taylor [41], but that argument does not generalize to this case. Nonetheless, we feel that Conjecture 3.2 is a most reasonable conjecture to make.

The automorphic forms arising in Conjecture 3.2 are naturally associated to cohomology classes on their associated symmetric spaces, which in this case are 3-manifolds. We now recall the adelic construction of these manifolds. Fix a quadratic imaginary field $K$, which for ease of exposition we assume to have class number one. Let $\mathcal{O}:=\mathcal{O}_{K}$ be the ring of integers of $K$. Let $\mathfrak{p}, \mathfrak{q}$ be two distinct prime ideals of $\mathcal{O}$. Let $U$ be the open compact subgroup of $\mathrm{GL}_{2}\left(\mathbb{A}_{K}^{\infty}\right)$ such that

(1) $U_{v}$ is $\mathrm{GL}_{2}\left(\mathcal{O}_{v}\right)$ for all $v$ outside $\mathfrak{p}$ and $\mathfrak{q}$.

(2) $U_{\mathfrak{p}}$ is the set of matrices in $\mathrm{GL}_{2}\left(\mathcal{O}_{\mathfrak{p}}\right)$ of the form

$$
\left(\begin{array}{ll}
* & * \\
0 & *
\end{array}\right) \quad \bmod \mathfrak{p} .
$$

(3) $U_{\mathfrak{q}}$ is the set of matrices in $\mathrm{GL}_{2}\left(\mathcal{O}_{\mathfrak{q}}\right)$ of the form

$$
\left(\begin{array}{ll}
1 & * \\
0 & 1
\end{array}\right) \quad \bmod \mathfrak{q}^{n}
$$

Define

$$
X\left(\Gamma_{0}(\mathfrak{p}) \cap \Gamma_{1}\left(\mathfrak{q}^{n}\right)\right)=\mathrm{GL}_{2}(K) \backslash\left(\mathrm{GL}_{2}\left(\mathbb{A}_{K}^{\infty}\right) / U\right) \times \mathbb{M}^{3}
$$

Now let $D / K$ be the quaternion algebra ramified exactly at $\{\mathfrak{p}, \mathfrak{q}\}$. Let $B$ be a maximal order of $D$. Let $\mathfrak{m}$ be a maximal bi-ideal of $B$ that is trivial away from $\mathfrak{q}$. Let $V$ be the compact subgroup of $\mathrm{GL}_{2}\left(\mathbb{A}_{K}^{\infty}\right)$ that is $\left(1+\mathfrak{m}^{n}\right)_{v}$ for each $v$ (note, this will equal $B_{v}^{\times}$for all $\left.v \neq \mathfrak{q}\right)$. Define

$$
X\left[\mathfrak{p q}^{n}\right]=D^{\times} \backslash\left(\mathrm{GL}_{2}\left(\mathbb{A}_{K}^{\infty}\right) / V\right) \times \mathbb{M}^{3}
$$


The 3-orbifolds $X\left(\Gamma_{0}(\mathfrak{p}) \cap \Gamma_{1}\left(\mathfrak{q}^{n}\right)\right)$ and $X\left[\mathfrak{p q}{ }^{n}\right]$ have more concrete descriptions as arithmetic quotients of $\mathbb{M}^{3}$; the former as $\mathbb{\boxplus}^{3} /\left(U \cap \mathrm{GL}_{2}\left(\mathcal{O}_{K}\right)\right)$ and the latter as

$$
\mathbb{H}^{3} /\left(B^{\times} \cap\left(1+\mathfrak{m}^{n}\right)\right) \text {. }
$$

There is a well known correspondence between the cohomology of the 3-manifolds constructed above and automorphic forms (see for example Taylor [40, Section 4] or Harder [17]). A classical version of this association relates $H_{\text {cusp }}^{1}$ of modular curves to classical modular cusp forms. Recall here that for a manifold $X$ with boundary, the cuspidal cohomology $H_{\text {cusp }}^{1}(X ; \mathbb{C})$ is the kernel of the natural homomorphism

$$
H^{1}(X ; \mathbb{C}) \rightarrow H^{1}(\partial X ; \mathbb{C}) .
$$

The cuspidal cohomology of $X\left(\Gamma_{0}(\mathfrak{p}) \cap \Gamma_{1}\left(\mathfrak{q}^{n}\right)\right)$ is exactly associated to spaces of automorphic forms $\pi$ that satisfy the conditions of Conjecture 3.2, and thus we may study the cohomology by studying the associated (predicted) Galois representations. The cohomology of the compact manifold $X\left[\mathfrak{p q}^{n}\right]$ can also be associated to automorphic forms. The main theorem of Jacquet-Langlands [22, Section 16] implies that the resulting space forms will correspond in a precise way to a subset of the automorphic forms arising from $H_{\text {cusp }}^{1}\left(X\left(\Gamma_{0}(\mathfrak{p}) \cap \Gamma_{1}\left(\mathfrak{q}^{n}\right)\right) ; \mathbb{C}\right)$. Thus we obtain the following result, which remains completely mysterious from a topological point of view:

\subsection{Theorem (Jacquet-Langlands) If $H^{1}\left(X\left[\mathfrak{p q}^{n}\right]\right.$; $\left.\mathbb{C}\right)$ is non-zero then}

$$
H_{\text {cusp }}^{1}\left(X\left(\Gamma_{0}(\mathfrak{p}) \cap \Gamma_{1}\left(\mathfrak{q}^{m}\right)\right) ; \mathbb{C}\right) \quad \text { is non-zero for some } m \leq 2 n .
$$

A more precise version of this theorem relates not only these cohomology groups as $\mathbb{C}$-vector spaces but also as modules for the action of the so called Hecke operators (namely, the correspondence preserves eigenspaces and eigenvalues). However, we only apply this result to conclude that the former cohomology group vanishes because the latter one does, and so we suppress this extra detail. In particular, the conclusion of this theorem that we will need is the following:

3.4 Theorem Assume Conjecture 3.2. Suppose that $H^{1}\left(X\left[\mathfrak{p q}^{n}\right] ; \mathbb{Q}\right) \neq 0$. Suppose that $\mathfrak{p}$ has residual characteristic $p$. Then there exists a field $\left[E: \mathbb{Q}_{p}\right]<\infty$ and a continuous Galois representation $\rho: \operatorname{Gal}(\bar{K} / K) \rightarrow \mathrm{GL}_{2}(E)$ such that

(1) $\rho$ is ordinary at $\mathfrak{p}$,

(2) $\rho$ is unramified outside $\mathfrak{q}$ and primes dividing $p$.

(3) $\operatorname{det}(\rho)=\chi \psi$, where $\psi$ is a finite character unramified outside $\mathfrak{q}$.

(4) $\rho$ is irreducible. 
Proof By the universal coefficient theorem, if $\mathbb{Q}$-cohomology is trivial then so is $\mathbb{C}$-cohomology; hence $H^{1}\left(X\left[\mathfrak{p q}^{n}\right] ; \mathbb{C}\right) \neq 0$. By Jacquet-Langlands, the non-triviality of this cohomology implies that $H_{\text {cusp }}^{1}\left(X\left(\Gamma_{0}(\mathfrak{p}) \cap \Gamma_{1}\left(\mathfrak{q}^{m}\right)\right) ; \mathbb{C}\right)$ is also nonzero, for some $m$. As mentioned above, the non-vanishing of this cuspidal cohomology gives an automorphic representations $\pi$ which satisfies the hypotheses of Conjecture 3.2. The Galois representation produced by this conjecture then satisfies (1)-(4).

Theorem 1.6 may be restated in the language of this section as follows:

3.5 Theorem Assume Conjecture 3.2 and the GRH. Let $K=\mathbb{Q}(\sqrt{-2})$, and let $3=\pi \bar{\pi}$ in $\mathcal{O}_{K}$. Then $H^{1}\left(X\left[\bar{\pi} \pi^{n}\right] ; \mathbb{Q}\right)=0$ for all $n$.

Taking $\mathfrak{p}=\bar{\pi}$ and $\mathfrak{q}=\pi$ in Theorem 3.4, we see that if $X\left[\bar{\pi} \pi^{n}\right]$ has nontrivial $H^{1}$ then there exists a 3-adic Galois representations satisfying properties (1)-(4). Thus it suffices to prove that no such representations exist. This is exactly the content of Theorem 5.1, where it is shown that such a Galois representation satisfying (1)-(3) must violate (4). We complete the proof of this purely arithmetic result in the next two sections of this paper.

\section{Residual representations of small Serre level}

As a warm up to proving Theorem 5.1, we first study the possible residual parts of the Galois representations in question. Let $\mathbb{F}$ be a finite field of residue characteristic $p$. Let $K$ be a number field, and

$$
\bar{\rho}: \operatorname{Gal}(\bar{K} / K) \rightarrow \mathrm{GL}_{2}(\mathbb{F})
$$

a semisimple Galois representation of Serre conductor $N(\bar{\rho})$. A theorem of Tate [39] shows that if $(K, p, N(\bar{\rho}))=(\mathbb{Q}, 2,1)$ then $\bar{\rho}$ is trivial. We prove the following variant of Tate's results over an imaginary quadratic field.

4.1 Theorem Suppose that $(K, p, N(\bar{\rho}))=(\mathbb{Q}(\sqrt{-2}), 3,1)$. Let $3=\pi \bar{\pi}$ and suppose furthermore that $\operatorname{det}(\bar{\rho})=\chi$, the cyclotomic character restricted to $K$. Then assuming the GRH, $\bar{\rho}=\chi \oplus 1$.

Proof Without loss of generality we assume that $\bar{\rho}$ cannot be conjugated so that its image lies in $\mathrm{GL}_{2}\left(\mathbb{F}^{\prime}\right)$ for some proper subfield $\mathbb{F}^{\prime} \subset \mathbb{F}$. We shall break the proof up into three cases. Either

(1) The representation $\bar{\rho}$ is reducible. 
(2) The representation $\bar{\rho}$ is solvable but not reducible.

(3) The image of $\bar{\rho}$ is non-solvable.

If $\bar{\rho}$ is reducible and semisimple it breaks up as the direct sum of two characters:

$$
\eta, \eta^{\prime}: \operatorname{Gal}(\bar{K} / K) \rightarrow \mathbb{F}^{\times} .
$$

If $L$ is the maximal abelian extension of $K$ unramified outside 3 of order coprime to 3 , then by class field theory $\operatorname{Gal}(L / K)$ is isomorphic to $(\mathcal{O} / 3 \mathcal{O})^{\times} \simeq \mathbb{F}_{3}^{\times} \times \mathbb{F}_{3}^{\times}$ modulo $\mathcal{O}^{\times}=\{ \pm 1\}$. Since this quotient has order 2 , it follows that $L=K\left(\zeta_{3}\right)$. Since $\operatorname{det} \bar{\rho}=\chi$ it follows that $\eta$ and $\eta^{\prime}$ are (possibly after reordering) the trivial and cyclotomic character respectively.

If $\bar{\rho}$ is solvable and irreducible then the image of

$$
\widetilde{\rho}: \operatorname{Gal}(\bar{K} / K) \rightarrow \operatorname{PGL}_{2}(\mathbb{F})
$$

is either dihedral, $A_{4}$ or $S_{4}$. Since $\operatorname{det} \bar{\rho}=\chi$ the image of $\operatorname{det} \widetilde{\rho}$ is $\mathbb{F}_{3}^{\times} / \mathbb{F}_{3}^{\times 2} \simeq \mathbb{Z} / 2 \mathbb{Z}$. Since $A_{4}$ has no such quotients it follows that the image of $\widetilde{\rho}$ is either dihedral or $S_{4}$. Let $M / K$ be the field cut out by the kernel of $\widetilde{\rho}$.

We first consider the case where $M / K$ is dihedral. Suppose the degree $[M: K]$ is divisible by 3 . Then the image of $\bar{\rho}$ contains an element of order 3 , and it follows that the image of $\bar{\rho}$ lands inside a Borel subgroup of $\mathrm{GL}_{2}(\mathbb{F})$ (and is thus reducible) or contains $\mathrm{SL}_{2}\left(\mathbb{F}^{\prime}\right)$ for some subfield $\mathbb{F}^{\prime} \subset \mathbb{F}$. The former case has already been considered, and in the latter case the projective image $\widetilde{\rho}$ is either non-solvable or $S_{4}$. Thus 3 does not divide the order of [ $M: K]$. Let $L$ be the maximal extension of $K$ inside $M$ such that $L / K$ is abelian. Since $[L: K]$ is coprime to 3 , we find (as in the reducible case) that $\operatorname{Gal}(L / K)$ is a quotient of the cokernel $\{ \pm 1\} \rightarrow(\mathcal{O} / 3 \mathcal{O})^{\times} \simeq \mathbb{F}_{3}^{\times} \times \mathbb{F}_{3}^{\times}$. Since this cokernel has order 2 , it follows that $L \subseteq K\left(\zeta_{3}\right)=\mathbb{Q}(\sqrt{-2}, \sqrt{-3})$. The extension $M / L$ is abelian since $\widetilde{\rho}$ has dihedral image. Suppose that $M / L$ was ramified at some prime above 3 . Then $M / K$ is totally ramified at this prime, and thus the inertia group of $\operatorname{Gal}(M / K)$ is non-abelian, and has order coprime to 3. Yet the maximal tame quotient of inertia is pro-cyclic, and thus $M / L$ must be unramified everywhere. Since CL $(\mathbb{Q}(\sqrt{-2}, \sqrt{-3}))=1$, this is impossible.

Now assume that $M / K$ is an $S_{4}$-extension. Let $L$ be the maximal extension of $K$ inside $M$ such that $L / K$ is abelian. Since $\operatorname{Gal}(M / K)=S_{4}$, it follows that $\operatorname{Gal}(L / K)=\mathbb{Z} / 2 \mathbb{Z}$, and $([L: K], 3)=1$. Thus as above $L=K\left(\zeta_{3}\right)=\mathbb{Q}(\sqrt{-2}, \sqrt{-3})$. Let $J$ be the maximal abelian extension of $L$ contained in $M$. Then $\operatorname{Gal}(J / K)=S_{3}$. The only $S_{3}$ extensions of $K=\mathbb{Q}(\sqrt{-2})$ unramified outside 3 and containing $L$ are 
$L(\sqrt[3]{\pi}), L(\sqrt[3]{\bar{\pi}})$ and $L(\sqrt[3]{3})$. All these extensions are totally ramified over $K$ at both primes above 3 . Consider the inertia subgroup at a prime above 3 of $\operatorname{Gal}(M / K)$. It is a subgroup of $S_{4}$ that surjects onto $S_{3}$, and thus is either $S_{3}$ or $S_{4}$. Since $S_{4}$ cannot occur as the inertia group of an extension of $\mathbb{Q}_{3}$, it follows that $M / L$ is unramified at all primes above 3 and thus unramified everywhere. Yet the class number of each of the three prospective fields $L$ is one, and thus $M$ does not exist.

Finally, let us assume that the image of $\bar{\rho}$ is non-solvable. We will need the following lemma.

4.2 Lemma Let $\mathbb{F}$ be a finite field of characteristic $p$, and let

$$
\rho: \operatorname{Gal}(\bar{K} / K) \rightarrow \mathrm{GL}_{2}(\mathbb{F})
$$

be a continuous Galois representation. Fix a prime $\mathfrak{p}$ above $p$ in $K$. Then either

(1) The image of inertia at $\mathfrak{p}$ is tame.

(2) The image of the decomposition group at $\mathfrak{p}$ is reducible.

The proof of the lemma is a standard application of the fact that $p$-groups do not act freely on finite dimensional $\mathbb{F}_{p}$-vector spaces, and we leave it to the reader.

Let us consider the restriction of $\bar{\rho}$ to inertia at $\pi$. If the image of inertia under $\bar{\rho}$ has order coprime to 3 , then the largest power of $\pi$ dividing $\Delta_{L / K}$ is $\pi^{[L: K]-1}$. The contribution to the root discriminant $\delta_{L / \mathbb{Q}}=\left|\Delta_{L / \mathbb{Q}}\right|^{1 /[L: \mathbb{Q}]}$ is thus bounded above by $3^{1 / 2}$. Suppose the image of the decomposition group at $\pi$ is reducible. All characters $\operatorname{Gal}\left(\overline{\mathbb{Q}}_{3} / \mathbb{Q}_{3}\right) \rightarrow \mathbb{F}^{\times}$are the product of an unramified character and a power of the cyclotomic character. Thus the tame quotient has order 2 , and the image of wild inertia is contained in the set of matrices of the form

$$
\left(\begin{array}{ll}
1 & * \\
0 & 1
\end{array}\right) \subset \mathrm{GL}_{2}(\mathbb{F})
$$

This subgroup is isomorphic to $\mathbb{F}$. In particular, it is an elementary three group of order $3^{m}=\|\mathbb{F}\|$. An explicit calculation (see Tate [39]) now shows that this is the largest possible power of $\pi$ dividing $\Delta_{L / K}$ is $\left(2+1 / 3-1 / 2 \cdot 3^{1-m}\right)[L: K]$. A similar calculation also applies to $\bar{\pi}$. Thus if $3^{m}=\|\mathbb{F}\|$, then the root discriminant $\delta_{L / \mathbb{Q}}$ is bounded as follows:

$$
\log \left(\delta_{L / \mathbb{Q}}\right) \leq \log \left(\delta_{K / \mathbb{Q}}\right)+\log (3)\left(2+1 / 3-1 / 2 \cdot 3^{1-m}\right) .
$$

On the other hand, since $\operatorname{det} \bar{\rho}=\chi$, we know that $L$ contains $\mathbb{Q}(\sqrt{-2}, \sqrt{-3})$. Let $G=\operatorname{Gal}(L / K)$ and $H=\operatorname{Gal}(L / \mathbb{Q}(\sqrt{-2}, \sqrt{-3}))$. Since $G$ is non-solvable the 
image of $G$ in $\mathrm{PGL}_{2}(\mathbb{F})$ is either $A_{5}$ or contains the group $\mathrm{PSL}_{2}(\mathbb{F})$ (see Serre [38, Lemma 2]). Since $\operatorname{det} \bar{\rho}=\chi$ the image of $\operatorname{det} \widetilde{\rho}$ is $\mathbb{F}_{3}^{\times} / \mathbb{F}_{3}^{\times 2} \simeq \mathbb{Z} / 2 \mathbb{Z}$. Since $A_{5}$ admits no such quotient we see that the image of $G$ must be all of $\operatorname{PGL}_{2}(\mathbb{F})$. It follows that the image of $H$ is all of $\mathrm{PSL}_{2}(\mathbb{F})$, and thus by a classification of subgroups of $\mathrm{SL}_{2}(\mathbb{F})$ that $H=\mathrm{SL}_{2}(\mathbb{F})$. In particular,

$$
[L: \mathbb{Q}]=\# H \cdot[\mathbb{Q}(\sqrt{-2}, \sqrt{-3}): \mathbb{Q}]=4\left\|\mathrm{SL}_{2}(\mathbb{F})\right\|=4\left(3^{2 m}-1\right) 3^{m} .
$$

On the other hand, the GRH discriminant bounds of Odlyzko [29] imply that for sufficiently small $\delta_{L / \mathbb{Q}}$ the degree $[L: \mathbb{Q}]$ is bounded above. Letting $B$ denote this upper bound for various values of $\delta_{L / \mathbb{Q}}$ we have the following table:

\begin{tabular}{c|c|c|c}
$m$ & Upper bound on $\delta_{L / \mathbb{Q}}$ from (4.3) & $B$ & $4\left\|\mathrm{SL}_{2}(\mathbb{F})\right\|$ \\
\hline 2 & $2^{3 / 2} 3^{13 / 6}=30.5708639321$ & 2400 & 2880 \\
3 & $2^{3 / 2} 3^{41 / 18}=34.5399086640$ & 10000 & 78624 \\
$4 \ldots \infty$ & $2^{3 / 2} 3^{7 / 3}=36.7136802477$ & 100000 & $\geq 2125440$
\end{tabular}

For each possible value of $m$, we have $[L: \mathbb{Q}]=4\left\|\mathrm{SL}_{2}(\mathbb{F})\right\|>B \geq[L: \mathbb{Q}]$, a contradiction. It follows (on the GRH) that $\bar{\rho}$ must have solvable image, and thus by previous considerations must be $\chi \oplus 1$.

4.5 Remark We note that there is a plausible technique for removing the assumption on the GRH. If we assume that $\bar{\rho}$ is modular (which is sufficient for our applications), then a generalized Serre's conjecture would imply that $\bar{\rho}$ arises from a mod-3 modular form of sufficiently small level and weight which can then be computed explicitly. However, we prefer to assume the GRH conjecture, since although it is probably more difficult, it has the benefit of already being widely considered. In contrast, possible generalizations of level lowering that may as yet reveal unexpected phenomena.

\section{Global representations of small conductor}

As discussed at the end of Section 3, in order to complete the proof of Theorem 1.6 it suffices to show:

5.1 Theorem Let $K=\mathbb{Q}(\sqrt{-2})$, and let $3=\pi \bar{\pi}$ in $\mathcal{O}_{K}$. Let $E$ be a local field of mixed residue characteristic 3 . Let

$$
\rho: \operatorname{Gal}(\bar{K} / K) \rightarrow \mathrm{GL}_{2}(E)
$$

be a continuous Galois representation such that 
(1) $\rho$ is ordinary at $\bar{\pi}$.

(2) $\rho$ is unramified outside $\pi$ and $\bar{\pi}$.

(3) $\operatorname{det}(\rho)=\chi \psi$, where $\psi$ is a finite character unramified outside $\pi$.

Then $\rho$ is reducible.

Proof By choosing a lattice in $E$ we may assume that $\rho$ has an integral representation $V$. Note that such a choice is not necessarily unique. Let $\mathbb{F}=\mathcal{O}_{E} / \mathfrak{m}_{E}$ be the (finite) residue field of characteristic three. The fixed field of $\bar{\psi}$ is an extension of $K$ of degree coprime to 3 unramified outside $\pi$. By class field theory, such extensions are classified by $(\mathcal{O} / \pi \mathcal{O})^{\times} \simeq \mathbb{F}_{3}^{\times}$modulo global units. Since the image of -1 generates $\mathbb{F}_{3}^{\times}$, it follows that $\psi$ is trivial modulo 3 . Thus $\bar{\rho}$ has determinant $\chi$, and hence by Theorem 4.1, the semisimplification $\bar{\rho}^{s s}$ is isomorphic to $\chi \oplus 1$. Thus $V$ has a filtration by modules isomorphic to $\mathbb{Z} / 3 \mathbb{Z}$ or $\mu_{3}$. By assumption $\rho$ is ordinary at $\bar{\pi}$. Thus there exists a filtration

$$
0 \rightarrow V^{\prime} \rightarrow V \rightarrow V^{\prime \prime} \rightarrow 0
$$

of $\operatorname{Gal}\left(\bar{K}_{\pi} / K_{\pi}\right)=\operatorname{Gal}\left(\overline{\mathbb{Q}}_{3} / \mathbb{Q}_{3}\right)$-modules where $V^{\prime} \otimes \mathbb{Q}$ and $V^{\prime \prime} \otimes \mathbb{Q}$ are free of rank one over $E$, and the filtered pieces of $V^{\prime} / p^{n}$ (respectively $V^{\prime \prime} / p^{n}$ ) are all isomorphic as $\operatorname{Gal}\left(\overline{\mathbb{Q}}_{3} / \mathbb{Q}_{3}\right)$-modules to $\mu_{3}$ (resp. $\left.\mathbb{Z} / 3 \mathbb{Z}\right)$. It therefore suffices to show that $V^{\prime} / p^{n}$ and $V^{\prime \prime} / p^{n}$ extend to $\operatorname{Gal}(\bar{K} / K)$-modules. Assume otherwise. Then there must exist an extension class in $\operatorname{Ext}^{1}\left(\mu_{3}, \mathbb{Z} / 3 \mathbb{Z}\right)$ that splits completely at $\bar{\pi}$. It thus suffices to prove the following:

5.2 Lemma The group of extensions $\operatorname{Ext}^{1}\left(\mu_{3}, \mathbb{Z} / 3 \mathbb{Z}\right)$ that are unramified outside $\pi$ and split completely at $\bar{\pi}$ are trivial.

Proof Any such Galois extension defines a $\chi^{-1}=\chi$ extension of $K\left(\zeta_{3}\right)$. Such extensions are of the form $L=K\left(\zeta_{3}, \gamma^{1 / 3}\right)$ where $\gamma \in K$. Moreover, $L / K$ is totally split at $\bar{\pi}$ if and only if one can take $\gamma \equiv 1 \bmod \bar{\pi}^{2}$. Since $L / K$ is unramified outside $\pi$ and $\operatorname{CL}\left(\mathcal{O}_{K}\right)=1$ it must be the case that $\gamma= \pm \pi$. Yet $\pm \pi \equiv \pm 4 \bmod \bar{\pi}^{2}$, and thus this extension does not split completely at $\bar{\pi}$ (indeed it is ramified at $\bar{\pi}$ ).

Having established the lemma, we've proven the theorem, and, as a consequence, completed the proof of Theorem 1.6.

We note the following corollary of Theorems 5.1 and 4.1.

5.3 Corollary Assuming the GRH, there does not exist an abelian variety of $\mathrm{GL}_{2}-$ type over $K=\mathbb{Q}(\sqrt{-2})$ with good reduction outside the prime $\pi=1-\sqrt{-2}$. 
Proof Consider such a variety $A$, and let $\rho$ be an associated $\mathrm{GL}_{2}$-representation occurring inside the 3-adic Tate module of $A$. By Theorem 4.1 the representation $\bar{\rho}$ is reducible. Since $\mathbb{Z} / 3 \mathbb{Z}$ and $\mu_{3}$ are the only finite flat group schemes of order 3 over $\operatorname{Spec}(\mathcal{O}[1 / \pi])$ it follows that $A$ has good ordinary reduction at $\bar{\pi}$. From Theorem 5.1 we conclude that $\rho$ is reducible. It follows that $A$ has CM by some order in $\mathcal{O}$. In particular, $A$ arises from base change from some totally real field, and thus from $\mathbb{Q}$. This implies that $A$ has good reduction at $\pi$, and thus good reduction everywhere over $\mathcal{O}$. The result then follows from Schoof [37].

\section{The Boston-Ellenberg approach: pro-p groups}

As mentioned in the introduction, Boston and Ellenberg [2] were able to improve Theorem 1.6 to an unconditional statement, not dependent on the GRH and Conjecture 3.2 ; their approach was to analyze the $M_{n}$ using the theory of pro- $p$ groups rather than automorphic forms and Galois representations. In this section, we will generalize their approach slightly so that it applies, as we illustrate, to a range of examples, both arithmetic and non-arithmetic.

In order to state the main result, we first need to give a number of definitions. To start, if $M$ is a hyperbolic 3-manifold, we say a tower of finite covers

$$
M_{0} \leftarrow M_{1} \leftarrow M_{2} \leftarrow M_{3} \leftarrow \cdots
$$

exhausts $M$ if $\operatorname{injrad}\left(M_{n}\right) \rightarrow \infty$ as $n \rightarrow \infty$. If $\beta_{1}\left(M_{n}\right)=H^{1}\left(M_{n} ; \mathbb{Q}\right)=0$ for all $n$, then we say $M$ can be exhausted by rational homology spheres. On a different note, any hyperbolic 3-manifold $M$, arithmetic or not, has an associated quaternion algebra $A_{0}(M)$, see eg Maclachlan-Reid [28, Chapter 3.3]. Here we are using the non-invariant quaternion algebra, which can change a little under finite covers.

Turning now to finite groups, the crucial definition is:

6.1 Definition Let $p$ be an odd prime. A finite $p$-group $S$ is powerful if $S / S^{p}$ is abelian, where $S^{p}$ is the subgroup generated by all $p^{\text {th }}$ powers. For $p=2, S$ is powerful if $S / S^{4}$ is abelian.

One should think of powerful $p$-groups as close to being abelian, and hence sharing many of the properties of abelian groups. For general groups, the following definition is central to our criterion:

6.2 Definition Let $G$ be a finitely generated group. We say that $G$ is $p$-powerful if every $p$-group quotient of $G$ is powerful. 
As we will explain in Remark 6.5, it is straightforward to check from a presentation whether $G$ is $p$-powerful. The main result of this section is:

6.3 Theorem Let $M$ be a hyperbolic 3-manifold whose quaternion algebra ramifies at a prime of norm $p^{n}$ where $p \in \mathbb{Z}$ is prime. Suppose that $\beta_{1}(M)=0$ and that $\left|H_{1}(M ; \mathbb{Z})\right|$ is coprime to $p^{2 n}-1$. If $\pi_{1}(M)$ is $p$-powerful then $M$ can be exhausted by rational homology spheres.

We will actually prove somewhat more than this, but the above is the one that is easy to apply in practice (see Section 6.7). It follows easily from the next proposition; we thank Alex Lubotzky for pointing out this formulation, which removes some unnecessary restrictions in our original version.

6.4 Proposition Let $G$ be a finitely generated group which is $p$-powerful. If $\beta_{1}(G)=0$, then any $H \unlhd G$ of $p$-power index also has $\beta_{1}(H)=0$.

The proof of the proposition uses some fairly elementary aspects of the theory of pro- $p$ groups. However, in some cases one can reduce Theorem 6.3 down to a single non-trivial theorem about finite $p$-groups; we give that argument in Section 6.8. We refer the reader to the book by Dixon, du Sautoy, Mann and Segal [12] for the needed background about pro- $-p$ groups.

Proof of Proposition 6.4 For this proof, it is natural to replace $G$ and $H$ by their pro- $p$ completions $\widehat{G}$ and $\widehat{H}$; since $[G: H]=p^{n}$, the completion $\widehat{H}$ is a finite index open subgroup of $\widehat{G}$. Note $G$ has a surjective homomorphism onto $\mathbb{Z}$ if and only if $\widehat{G}$ has one to $\mathbb{Z}_{p}$, and the same for $H$. Thus it is enough to show that if $\widehat{H} \rightarrow \mathbb{Z}_{p}$ then so does $\widehat{G}$.

For the rest of this proof, we work exclusively with pro- $-p$ groups, and so drop the decorations from $\widehat{G}$ and $\widehat{H}$ and denote them simply $G$ and $H$. Now suppose that $H \rightarrow \mathbb{Z}_{p}$. It suffices to show that some quotient of $G$ surjects onto $\mathbb{Z}_{p}$, and we first exploit this to reduce to the case where $G$ is uniformly powerful and $H$ is isomorphic to $\mathbb{Z}_{p}^{d}$, for $d>0$.

Consider the maximal torsion-free abelian quotient of $H$ :

$$
1 \rightarrow K \rightarrow H \rightarrow \mathbb{Z}_{p}^{d} \rightarrow 0 .
$$

As $K$ is characteristic in $H$, it is normal in $G$, and so we can replace $(G, H)$ with $\left(G / K, H / K=\mathbb{Z}_{p}^{d}\right)$. As $G$ is powerful, the set $T$ of all torsion elements in $G$ is in fact a finite subgroup and $G / T$ is uniformly powerful; thus replacing $(G, H)$ with $(G / T, H /(T \cap H) \cong H)$ reduces to the desired case. 
Now $H=\mathbb{Z}_{p}^{d}$ is a uniformly powerful open subgroup of the uniformly powerful $G$. Uniformly powerful groups are in fact $p$-adic analytic groups, and we will make use of their associated Lie algebras. (For another pro- $p$ approach requiring less machinery, see [12, Chapter 4, Example 9].) Since $H$ is abelian, the Lie bracket on $L(H)$ is trivial. The inclusion induced monomorphism of the Lie algebras $L(H) \rightarrow L(G)$ then implies that the Lie bracket on $L(G)$ is trivial as well. This forces $G$ to be abelian and hence isomorphic to $\mathbb{Z}_{p}^{d}\left[12\right.$, Corollary 7.16]. Thus $G$ surjects onto $\mathbb{Z}_{p}$, as desired. $\square$

Proof of Theorem 6.3 Let $G=\pi_{1}(M)$. By Proposition 6.4, every normal subgroup of $G$ of $p$-power index has $\beta_{1}=0$. Thus to complete the proof, we need to show that $G$ is residually a $p$-group, or equivalently, that $M$ can be exhausted by regular covers of degree $p^{n}$. If $\mathfrak{p}$ is the given prime where $A_{0}(M)$ ramifies, we can consider the principal congruence covers $M\left(\mathfrak{p}^{n}\right)$ of $M$ of level $\mathfrak{p}^{n}$ just as in Section 2.5. The first of these covers $M \leftarrow M(\mathfrak{p})$ is cyclic of degree dividing $p^{2 n}-1$ (see Maclachlan-Reid [28, Theorem 6.4.3] for further details). The constraint on $H_{1}(M ; \mathbb{Z})$ forces this cover to be trivial. The remaining covers $M\left(\mathfrak{p}^{n}\right) \leftarrow M\left(\mathfrak{p}^{n+1}\right)$ all have degree dividing $p^{2}$. Just as before, these are regular covers of $M$ which exhaust it, completing the proof.

6.5 Remark To apply Theorem 6.3, we need to be able to check that a given finitely presented group is $p$-powerful. First, for a finite $p$-group $S$, consider the lower exponent $-p$ central series

$$
S=P_{0}(S) \triangleright P_{1}(S) \triangleright \cdots \triangleright P_{k}(S)=\langle 1\rangle \quad \text { where } P_{i+1}(S)=P_{i}(S)^{p}\left[P_{i}(S), S\right] .
$$

Here the successive quotients are isomorphic to $(\mathbb{Z} / p \mathbb{Z})^{n}$ and $k$ is called the exponent$p$ class of $S$. Any finitely presented group $G$ has a maximal $p$-quotient of exponent $-p$ class $k$; see Newman-O'Brien [31] for details and GAP [44] or Magma [9] for an implementation. Now suppose $G$ is a finitely presented group, and $S$ the maximal $p$-quotient of exponent $-p$ class 2 . We claim that $G$ is $p$-powerful if and only if $S$ is powerful; this follows by noting that if $Q$ is a non-powerful $p$-quotient of $G$, then $Q / P_{2}(Q)$ is a non-powerful quotient of $S$ (see the lemma below). Since $S$ is computable from a presentation from $G$ and checking if $S$ is powerful is easy since it is finite, we have the needed test.

We turn now to the $p$-group lemma just used.

6.6 Lemma Let $Q$ be a $p$-group. Then $Q$ is powerful if and only if $Q / P_{2}(Q)$ is powerful.

Proof First suppose $p$ is odd. Noting that $Q / P_{2}(Q)$ is powerful if and only if $\left(Q / Q^{p}\right) / P_{2}\left(Q / Q^{p}\right)$ 
is abelian, it suffices to show that if $T$ has exponent $p$ then $T$ is abelian if and only if $T / P_{2}(T)$ is. If $T / P_{2}(T)$ is abelian then $P_{2}(T) \geq[T, T]=P_{1}(T)$ as $T^{p}=1$. Thus the series stabilizes at $P_{1}(T)=[T, T]=1$, and $T$ is abelian, as needed.

If $p=2$, we can similarly reduce to showing that a group $T$ with exponent 4 is abelian if $T / P_{2}(T)$ is abelian. We proceed by induction on $|T|$. If $T$ has exponent 2 , it is abelian so assume that $T^{2}$ is a non-trivial subgroup. Then as $T$ is 2-group, $N=T^{2} \cap Z(T)$ is non-trivial. By induction, $G / N$ is abelian, and hence $N \geq[T, T]$. Thus commutators are all central which implies $\left[t_{1}^{2}, t_{2}^{2}\right]=\left[t_{1}, t_{2}\right]^{4}=1$ for $t_{i} \in T$; thus $T^{2}$ is abelian of exponent 2 . Then $[T, T] \leq T^{2}$ is as well, which means $\left[t_{1}^{2}, t_{2}\right]=\left[t_{1}, t_{2}\right]^{2}=1$. Thus $P_{2}(T)=1$ and $T$ is abelian as desired.

\subsection{Examples}

While the hypotheses of Theorem 6.3 are easy to check, one does not expect to find so many examples. To say that $\pi_{1}(M)$ is $p$-powerful implies that pro- $p$ completion is analytic, and in fact these two conditions are equivalent, up to passing to subgroups of finite index. Lubotzky has shown that if $\operatorname{dim} H_{1}\left(M ; \mathbb{F}_{p}\right)>3$ then the pro- $p$ completion of $\pi_{1}(M)$ is not analytic (see Lubotzky [25], and apply Theorem 1.2 as strengthened by Remark 1.4, to the combination of Lemma 5.1 and Lemma 1.1). In fact, because a pro- $p$ group generated by two elements is solvable and $\pi_{1}(M)$ is not, it is easy to see that Theorem 6.3 can only apply when $\operatorname{dim} H_{1}\left(M ; \mathbb{F}_{p}\right)=3$.

One example where Theorem 6.3 applies is the manifold $M_{2}$ from Section 2.5, and this was Boston and Ellenberg's original insight [2]. We searched the Hodgson-Weeks census [47] for manifolds where Theorem 6.3 applies. First, in order for $\pi_{1}(M)$ to have a non-cyclic pro- $p$ completion, it is necessary that $\operatorname{dim} H_{1}\left(M ; \mathbb{F}_{p}\right) \geq 2$. Among the 11,126 manifolds in the census there are 719 such pairs $(M, p)$ where $p$ is an odd prime, typically 3 or 5 . (Technical aside: we exclude $v 1539(5,1)$ which has $H_{1}(M ; \mathbb{Z})=\mathbb{Z}^{2}$ and hence has this property for all $p$.) Then we looked at the lower exponent $-p$ central series of $G=\pi_{1}(M)$ as discussed in Section 6.7. If we set $d_{k}=\operatorname{dim} P_{k}(G) / P_{k-1}(G)$ we observed an apparent dichotomy of behavior:

(1) The $d_{k}$ are uniformly bounded by 3. (61\% of the cases.)

(2) The $d_{k}$ eventually start to grow exponentially with $k$. (39\% of the cases.)

These should correspond precisely to whether the pro- $p$ completion of $G$ is analytic or not. In the former case, we confirmed this by checking that some $P_{k}(G)$ is in fact powerful (where $k \leq 2$ ) in all but one example when $p \leq 7$, but did not check the 37 examples where $p>7$. In the second case, that these groups must be non-analytic 


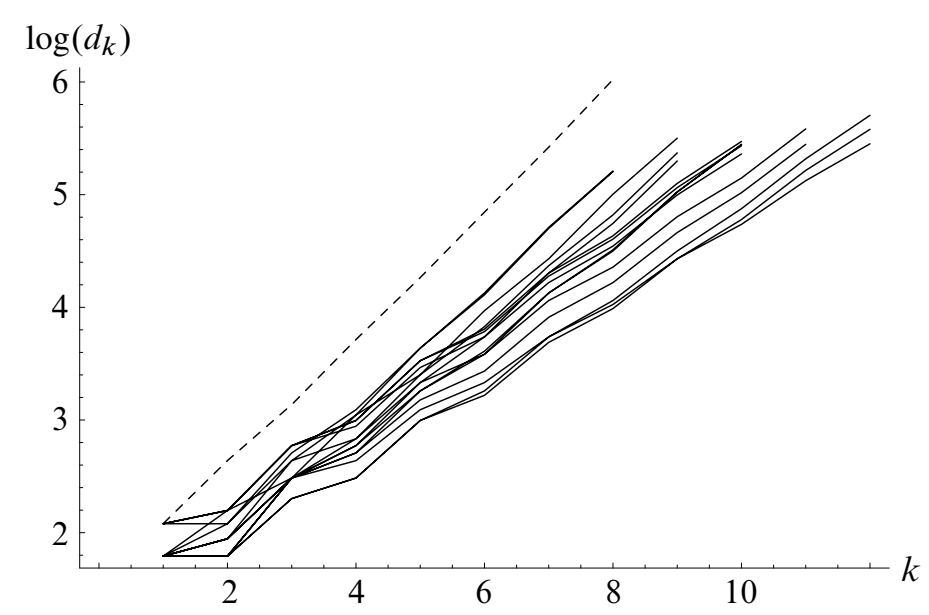

Figure 5: This figure shows the exponential growth of the $d_{k}$. The solid lines plot $\left(k-k_{0}, \log \left(d_{k-k_{0}}\right)\right)$ for each group, where $k_{0}$ is the first $k$ for which $d_{k}>5$ (that is, the first few small $d_{k}$ are omitted to save space). The dotted line is the same quantity for the free group of rank 2 . Even though this plot shows 278 different groups, the $d_{k}$ fall into only 15 distinct patterns.

follows from the result of Lubotzky mentioned above. The apparent exponential growth is clear from Figure 5. Most of these groups have 2 generators, and so for comparison it is interesting to consider the free group of rank 2. In that case, for any prime $p$, Witt's formula gives $d_{k}=\sum_{m=1}^{k}(1 / m) \sum_{d \mid m} \mu(m / d) 2^{d}$, which implies exponential growth at rate $\log 2$; that is $\lim _{k \rightarrow \infty}(1 / k) \log d_{k}=\log 2$. As you can see from Figure 5 , the non-analytic groups seem quite close to the free group in this sense.

Turning now to those manifolds where the pro- $p$ completion is analytic, we need to check the quaternion algebra hypothesis of Theorem 6.3, which can be done using the program Snap [10;15]. Unfortunately, Snap often fails to compute the needed information for the larger examples, so we content ourselves with simply listing 20 examples where Theorem 6.3 applies, as opposed to an exhaustive list. In all cases, the prime at which the quaternion algebra ramifies has norm a rational prime.

$$
\begin{aligned}
& p=3: \frac{s 649(-5,1), m 007(3,2), s 784(3,1), s 784(-5,1), v 2715(-1,2),}{v 3215(1,2), v 3215(5,1)}, \underline{m 285(1,2), v 2616(4,1), v 2674(-1,3)}, v 2933(1,4), \\
& p=5: m 003(-3,1), \underline{m 216(4,3), s 889(3,2), v 2739(1,2)}, \underline{m 017(-3,2),}, \underline{m 017(-5,1)}, \underline{m 022(-5,1)}, \underline{m 030(1,3)}, s 527(-3,2)
\end{aligned}
$$

Here the non-arithmetic examples are underlined. With the exception of the last two examples for $p=3$, the theorem is really applied to the cover corresponding to the 
kernel of $\pi_{1}(M) \rightarrow H_{1}\left(M ; \mathbb{F}_{p}\right)$. Also note that the first example for $p=5$ is the Weeks manifold.

We also looked at what happened for $p=2$. There are 1492 census manifolds where $\operatorname{dim} H^{1}\left(M ; \mathbb{F}_{2}\right) \geq 2$. The situation was similar to before, but a new behavior appears:

(1) The maximal $2-$ quotient of $\pi_{1}(M)$ is finite. (34\% of the cases.)

(2) The pro-2 completion of $\pi_{1}(M)$ is analytic and apparently infinite. (28\% of the cases.)

(3) The pro-2 completion of $\pi_{1}(M)$ is non-analytic and the $d_{i}$ appear to have an exponential growth rate $(0.25,0.55)$. (39\% of the cases.)

Here are 19 examples where Theorem 6.3 applies to the kernel of $\pi_{1}(M) \rightarrow H_{1}\left(M ; \mathbb{F}_{2}\right)$ :

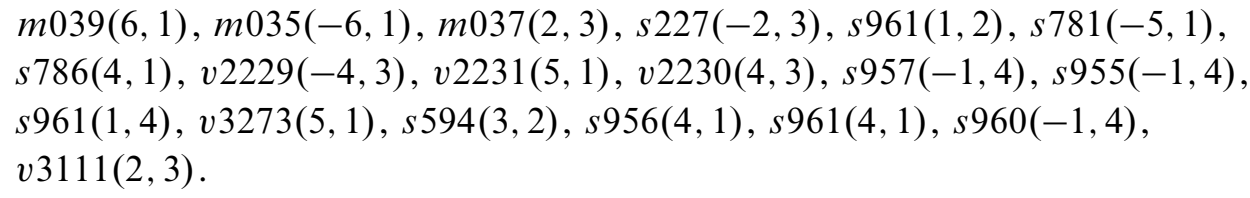

Here the first three are arithmetic, and all the others are non-arithmetic. Complete software (in Magma [9]) and data files for all of the above may be obtained at [5].

\subsection{Alternate approach}

It is possible to circumvent most of the pro- $p$ machinery used in the proof of Proposition 6.4 , at least if one increases the hypothesis somewhat. In this section we give a version which is enough to apply to many of the examples in the preceding section, yet relies on only a single theorem about $p$-groups.

Throughout we fix a prime $p$. For any group, let $d(S)=\operatorname{dim}\left(H^{1}\left(S ; \mathbb{F}_{p}\right)\right)$; for a $p$-group, this is equal to the minimal number of generators (see Dixon-du SautoyMann-Segal [12, Theorem 0.9]). Then one has the following basic fact about powerful $p$-groups, which is one of the ways they are analogous to abelian groups.

6.9 Theorem (Dixon-du Sautoy-Mann-Segal [12, page 41]) Let $S$ be a powerful $p$-group. If $H$ is any subgroup of $S$ then $d(H) \leq d(S)$.

Using this we will show

6.10 Proposition Let $G$ be a finitely presented group. Suppose $G$ is $p$-powerful for $p \geq 5$, and that $d(G) \leq 3$ and $\beta_{1}(G)=0$. If $N$ is a normal subgroup of $G$ of index $p^{n}$ then $\beta_{1}(N)=0$. 
As noted in Section 6.7, in when Theorem 6.3 applies one always has $d\left(\pi_{1}(M)\right) \leq 3$. Thus Proposition 6.10 can stand in for Proposition 6.4 in the proof of Theorem 6.3 whenever $p \geq 5$.

Proof First, we claim that $\beta_{1}(N) \leq 3$; this follows if we can show $d(N) \leq 3$. Consider the mod $-p$ abelianization map

$$
1 \rightarrow K \rightarrow N \rightarrow H_{1}\left(N ; \mathbb{F}_{p}\right) \rightarrow 0 .
$$

Then as $K$ is a characteristic subgroup of $N$, it is normal in $G$. By assumption, the $p$-group $G / K$ is powerful. Thus by the above theorem

$$
d(N)=d(N / K) \leq d(G / K) \leq d(G) \leq 3
$$

as desired.

To complete the proof, it suffices to show that $\beta_{1}(N)=\beta_{1}(G)$. Consider the action of $S=G / N$ on $H_{1}(N ; \mathbb{Z}) /($ torsion $)$. This gives us a homomorphism

$$
S \rightarrow \mathrm{GL}_{\beta_{1}(N)} \mathbb{Z} \leq \mathrm{GL}_{3} \mathbb{Z} .
$$

If this map is trivial, then one has an isomorphism between $H_{1}(N ; \mathbb{Q})$ and $H_{1}(G ; \mathbb{Q})$ as desired. But it is easy to see this map is trivial - just note that the only primes which are orders of elements of $\mathrm{GL}_{3} \mathbb{Z}$ are 2 and 3, since the characteristic polynomial of an element of $\mathrm{GL}_{3} \mathbb{Z}$ has degree 3 .

\section{Twist-knot orbifolds}

In this section, we investigate the congruence covers of twist-knot orbifolds. In contrast with the rest of the paper, our approach here is experimental - we simply examine large numbers of such covers. Interestingly, we find an apparent dichotomy of behavior between the arithmetic and non-arithmetic examples. In particular, the congruence covers of the arithmetic orbifolds were much more likely to have $\beta_{1}>0$.

Let us first roughly outline the results; a precise account follows. The twist knots $K_{n}$, for $n \in \mathbb{Z}$, are a simple family of knots described in Figure 6 . Here, $K_{1}$ is the trefoil, $K_{0}$ the unknot, and $K_{-1}$ the figure-8. We define $T(n, k)$ to be the orbifold whose underlying space is $S^{3}$, and singular set consists of the twist knot $K_{n}$ labeled by $k$. We focused on the $T(n, k)$ which are hyperbolic and where $|n| \leq 4$ and $3 \leq k \leq 7$; we examined 31 of these orbifolds. For each $T(n, k)$, we looked at congruence covers of the form $\Gamma_{0}(\mathfrak{p})$, which are covers of degree $N(\mathfrak{p})+1$. We did this for all $\mathfrak{p}$ of norm less than 10,000, which gave about 600-1200 covers for each $T(n, k)$. The natural 


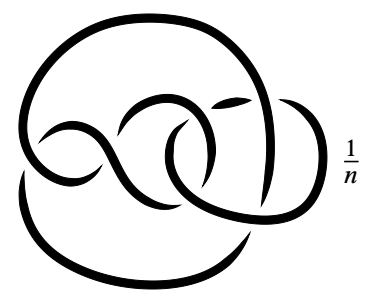

Figure 6: $K_{n}$ is obtained by doing $\frac{1}{n}$ Dehn surgery on the Whitehead link.

question is: what percentage of these covers have $\beta_{1}>0$ ? Keeping the base orbifold $T(n, k)$ fixed, we observed two distinct kinds of behavior

(1) A large proportion, $26-66 \%$ of the covers $\Gamma_{0}(\mathfrak{p})$ have $\beta_{1}>0$.

(2) Less than $2 \%$ of the covers have $\beta_{1}>0$.

About a third of the $T(n, k)$ fell into the first category, with the rest having the rare $\beta_{1}>0$ behavior. From a topological point of view, this dichotomy is rather odd since these manifolds are closely related (see in particular the formula (7.2) for $\pi_{1}$ ). Surprisingly, this dichotomy corresponds to exactly whether $T(n, k)$ is arithmetic, with the arithmetic manifolds falling into case (1). This table summarizes the data:

\begin{tabular}{rl|ccccccc} 
& \multicolumn{7}{c}{ Twist parameter $n$ in $K_{n}$} \\
\cline { 2 - 10 } & & -4 & -3 & -2 & -1 & 2 & 3 & 4 \\
\hline Orbifold & 3 & 0.9 & 49.0 & 55.5 & & 40.8 & 41.6 & 1.3 \\
label $k$ & 5 & 1.0 & 0.8 & 55.0 & 40.7 & 65.5 & 1.5 & 0.5 \\
& 5 & 1.1 & 0.7 & 0.8 & 54.8 & 56.8 & 0.9 & 0.9 \\
& 7 & 0.9 & 0.9 & 0.7 & 36.3 & 26.0 & 0.7 & 1.6 \\
& & & & & 2.0 & 1.7 & 0.4 & 1.1
\end{tabular}

Table 1: Percentage of $\Gamma_{0}(\mathfrak{p})$ with $\beta_{1}>0$, where $N(\mathfrak{p}) \leq 10,000$.

The shaded boxes are the arithmetic $T(n, k)$. Here, $T(0, k)$ and $T(-1,3)$ were omitted because they are not hyperbolic. The number of covers examined was in the range [578,632] when $k$ was even, and in $[1168,1247]$ when $k$ was odd. Which $T(n, k)$ are arithmetic was determined by Hilden-Lozano-Montesinos-Amilibia [20]. The arithmetic $T(n, k)$ all fall into the classes of such known to satisfy Conjecture 1.2. In a few cases, this is because the trace field has a subfield of index 2 and so the results of Labesse-Schwermer [24] and Lubotzky [26] applied, but in most cases one needs to use the result of Clozel [7]. 
For the non-arithmetic examples, it is worth listing the norms of the exceptional primes $\mathfrak{p}$ where $\beta_{1}\left(\Gamma_{0}(\mathfrak{p})\right)>0$ :

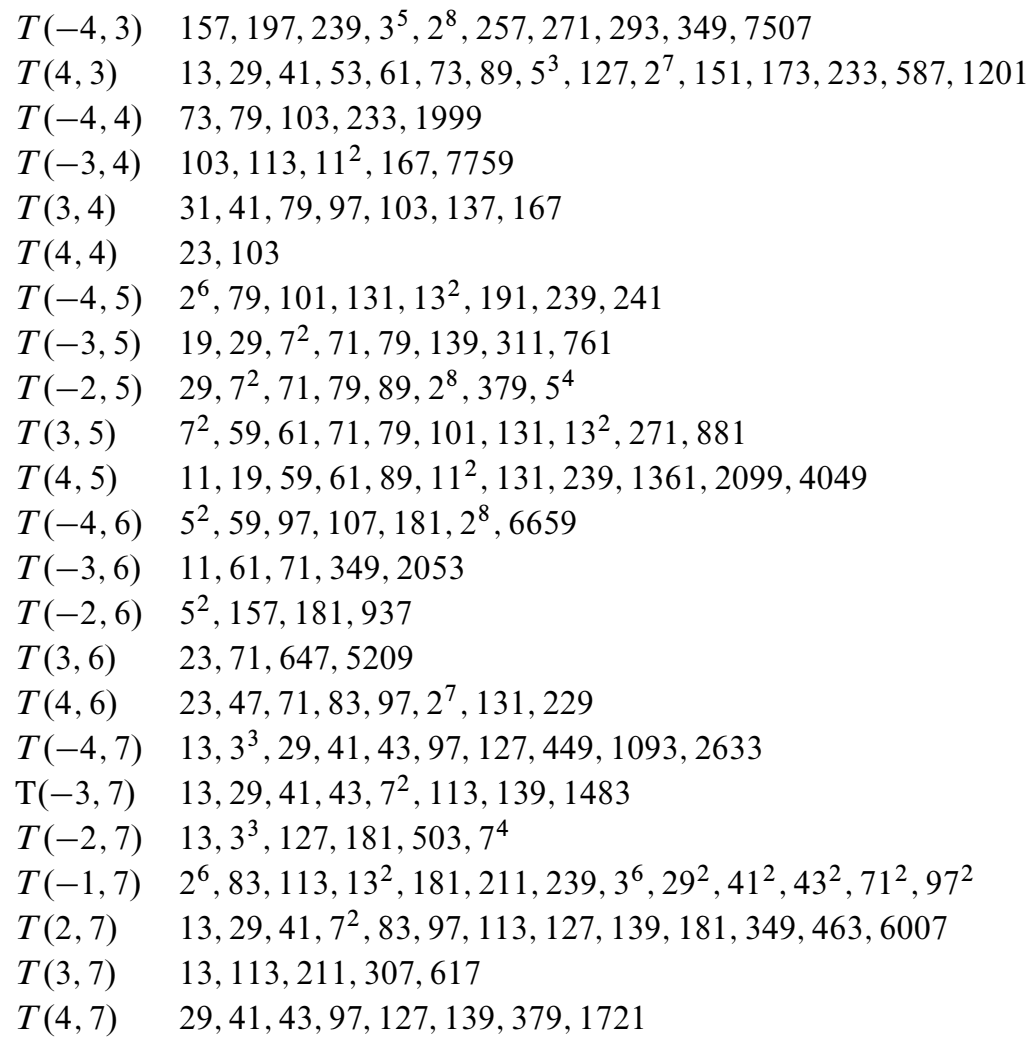

We examined two of the $T(n, k)$ more closely, computing additional covers out to $N(\mathfrak{p}) \leq 25,000$. The first of these is $T(4,4)$, which above has just 2 covers with $\beta_{1}>0$. This pattern continued; there were 727 covers $\Gamma_{0}(\mathfrak{p})$ with $N(\mathfrak{p})$ in $[10,000,25,000]$, and none of these had $\beta_{1}>0$. Should it be possible to answer Cooper's question from a topological or combinatorial viewpoint, $T(4,4)$ might be a good place to start. Indeed, it seems plausible that $T(4,4)$ has only finitely many congruence covers of the form $\Gamma_{0}(\mathfrak{p})$ which have $\beta_{1}>0$. Perhaps this is true of the principal congruence covers as well. In which case, trying to prove Conjecture 1.2 solely by looking an congruence covers would be a bad idea. The other one we looked at is $T(-1,7)$. In this case, the trend again continues, with $\beta_{1}\left(\Gamma_{0}(\mathfrak{p})\right)>0$ for a series of primes $\mathfrak{p}$ with square norm: $113^{2}, 127^{2}, 139^{2}$. Complete data, including the Magma source code used for the computations, can be found at the accompanying website for this paper [5]. 


\subsection{Details}

We now describe the $T(n, k)$ in more detail, explain exactly which covers we looked at, and give a few computational caveats. The orbifold fundamental group of $T(n, k)$ is given by:

$$
\Gamma=\left\langle a, b \mid a^{k}=b^{k}=1, w^{n} a=b w^{n}\right\rangle \quad \text { where } w=b a^{-1} b^{-1} a .
$$

Assuming $T(n, k)$ is hyperbolic, consider the representation $\rho_{0}: \Gamma \rightarrow \mathrm{PSL}_{2} \mathbb{C}$ corresponding to the hyperbolic structure. Regardless of whether $T(n, k)$ is arithmetic, Weil's local rigidity theorem [48] shows that $\rho$ can be conjugated so that the image lies in $\mathrm{PSL}_{2} L$, for some number field $L$. Explicitly,

$$
\rho_{0}(a)=\left(\begin{array}{cc}
e^{i \pi / k} & 1 \\
0 & e^{-i \pi / k}
\end{array}\right) \quad \rho_{0}(b)=\left(\begin{array}{cc}
e^{i \pi / k} & 0 \\
t & e^{-i \pi / k}
\end{array}\right)
$$

where $t$ is an algebraic integer satisfying a monic polynomial $r(z)$ in $\mathbb{Z}\left[e^{i \pi / k}\right]$ of degree $\approx 2|n|$ (see Hoste-Shanahan [21, Section 2]).

Let $L=\mathbb{Q}\left(e^{i \pi / k}, t\right)$ be the field generated by the entries of $\left\{\rho_{0}(\gamma) \mid \gamma \in \Gamma\right\}$, and let $K=\mathbb{Q}\left(e^{i \pi / k}+e^{-i \pi / k}, t\right)$ be the field generated by $\operatorname{tr}\left(\rho_{0}(\gamma)\right)$. Then $[L: K]$ is at most 2 , and indeed is 2 in the cases at hand. Let $\mathfrak{p}$ be a prime ideal of $\mathcal{O}_{K}$, and let $\mathfrak{q}$ be a prime ideal of $\mathcal{O}_{L}$ which divides $\mathfrak{p}$. Now consider the congruence quotient $\Gamma \rightarrow \operatorname{PSL}_{2}\left(\mathcal{O}_{L} / \mathfrak{q}\right)$. By definition, the congruence cover $\Gamma_{0}(\mathfrak{p})$ is the inverse image of a Borel subgroup under this map - that is, those $\gamma \in \Gamma$ which are upper-triangular $\bmod \mathfrak{q}$. The reason $\Gamma_{0}(\mathfrak{p})$ can be thought of as a function of $\mathfrak{p}$ rather than $\mathfrak{q}$ is that if the choice for $\mathfrak{q}$ is not unique, ie $\mathfrak{p}$ splits in $\mathcal{O}_{L}$, then the two choices for $\mathfrak{q}$ give congruence quotients with the same kernel. For all but finitely many $\mathfrak{p}$ which do split in $\mathcal{O}_{L}$, the homomorphism $\Gamma \rightarrow \operatorname{PSL}_{2}\left(\mathcal{O}_{L} / \mathfrak{q}\right)$ is surjective; in this case, $\Gamma_{0}(\mathfrak{p})$ has index $\left\|P^{1}\left(\mathcal{O}_{L} / \mathfrak{q}\right)\right\|=N(\mathfrak{p})+1$. For all but finitely many $\mathfrak{p}$ which do not split in $\mathcal{O}_{L}$, the homomorphism $\Gamma \rightarrow \operatorname{PSL}_{2}\left(\mathcal{O}_{L} / \mathfrak{q}\right)$ maps onto a conjugate of the smaller group $\operatorname{PSL}_{2}\left(\mathcal{O}_{K} / \mathfrak{p}\right)$. Again, $\Gamma_{0}(\mathfrak{p})$ has index $N(\mathfrak{p})+1$. (A more elegant point of view here would be construct these quotients by localizing the quaternion algebra associated to $\rho(\Gamma)$; this is also the connection between the definition here and the one described in Section 2.5.)

The reason we did not look at the principal congruence cover, which is the kernel of $\Gamma \rightarrow \operatorname{PSL}_{2}\left(\mathcal{O}_{L} / \mathfrak{q}\right)$, is purely pragmatic; because those covers are so much larger it's not possible to get beyond $N(\mathfrak{p})$ a few hundred, and so there would not be enough data to draw interesting conclusions. As it was, the computations took several CPU-months. 
We did not implement the above definition of $\Gamma_{0}(\mathfrak{p})$ directly because of the degrees of some of the fields involved. Instead, for each finite field $\mathbb{F}_{q}$, we searched directly for epimorphisms $\Gamma \rightarrow \mathrm{PSL}_{2} \mathbb{F}_{q}$, in a way that generates all the congruence quotients, as well as a possibly a few additional epimorphisms for small $\mathbb{F}_{q}$. Following Hilden-LozanoMontesinos-Amilibia [20] and Hoste-Shanahan [21], an irreducible representation $\pi_{1}(T(n, k)) \rightarrow \mathrm{PSL}_{2} E$, for any field $E$, is essentially determined by $\operatorname{tr}\left(\rho_{0}(a)^{2}\right)$ and $\operatorname{tr}\left(\rho_{0}(a b)\right)$. These quantities must satisfy certain integer polynomials, and conversely any solution gives a representation. Thus we simply searched for solutions to these equations over $\mathbb{F}_{q}$ to find the needed epimorphisms. However, one must be careful as the "trace variety" defined by these equations is not always irreducible over $\mathbb{Q}$. When it is reducible, we worked out the subvariety containing the image of the canonical representation $\rho_{0}$, and then used the equations defining that subvariety in our search. This ensures that only finitely many non-congruence covers are generated. When counting epimorphisms, we considered two epimorphisms $\Gamma \rightarrow \mathrm{PSL}_{2} \mathbb{F}_{q}$ equivalent if they different by an automorphism of $\operatorname{PSL}_{2}\left(\mathbb{F}_{q}\right)$; this differs from counting the number of ideals of $\mathcal{O}_{K}$ of norm $q$.

Finally, for speed reasons, when we determined whether $\beta_{1}>0$ we cheated a bit and worked over the finite field $\mathbb{F}_{31991}$ rather than $\mathbb{Q}$. Thus there could be some false positives where the cover really doesn't have $\beta_{1}>0$, though of course there are no false negatives. Based on the experience of Dunfield-Thurston [13], we expect there are at most a handful of such false positives, if any at all.

\section{References}

[1] M Baker, M Boileau, S Wang, Towers of covers of hyperbolic 3-manifolds, Rend. Istit. Mat. Univ. Trieste 32 (2001) 35-43 (2002) MR1893391

[2] N Boston, J S Ellenberg, Pro-p groups and towers of rational homology spheres, Geom. Topol. 10 (2006) 331-334

[3] A Brumer, K Kramer, Non-existence of certain semistable abelian varieties, Manuscripta Math. 106 (2001) 291-304 MR1869222

[4] F Calegari, Semistable abelian varieties over $\mathbb{Q}$, Manuscripta Math. 113 (2004) 507529 MR2129874

[5] F Calegari, N M Dunfield, Data files and Magma programs Available at http:// www. computop.org/software/twist-congruence/

[6] H Carayol, Sur les représentations l-adiques associées aux formes modulaires de Hilbert, Ann. Sci. École Norm. Sup. (4) 19 (1986) 409-468 MR870690

[7] L Clozel, On the cuspidal cohomology of arithmetic subgroups of $\operatorname{SL}(2 n)$ and the first Betti number of arithmetic 3-manifolds, Duke Math. J. 55 (1987) 475-486 MR894591 
[8] L Clozel, On the cohomology of Kottwitz's arithmetic varieties, Duke Math. J. 72 (1993) 757-795 MR1253624

[9] Computational Algebra Group, Magma, version 2.12 (2003) Available at http:// magma.maths.usyd.edu.au/

[10] D Coulson, O A Goodman, C D Hodgson, W D Neumann, Computing arithmetic invariants of 3-manifolds, Experiment. Math. 9 (2000) 127-152 MR1758805

[11] J E Cremona, Hyperbolic tessellations, modular symbols, and elliptic curves over complex quadratic fields, Compositio Math. 51 (1984) 275-324 MR743014

[12] J D Dixon, M P F du Sautoy, A Mann, D Segal, Analytic pro- $p$ groups, Cambridge Studies in Advanced Mathematics 61, Cambridge University Press, Cambridge (1999) MR1720368

[13] N M Dunfield, W P Thurston, The virtual Haken conjecture: experiments and examples, Geom. Topol. 7 (2003) 399-441 MR1988291

[14] J-M Fontaine, Il n'y a pas de variété abélienne sur $\mathbb{Z}$, Invent. Math. 81 (1985) 515-538 MR807070

[15] O Goodman, Snap: a computer program for studying arithmetic invariants of hyperbolic 3-manifolds Available at http://www.ms.unimelb.edu.au/ snap/

[16] F Grunewald, H Helling, J Mennicke, $\mathrm{SL}_{2}$ over complex quadratic number fields $I$, Algebra i Logika 17 (1978) 512-580, 622 MR555260

[17] G Harder, Eisenstein cohomology of arithmetic groups. The case $\mathrm{GL}_{2}$, Invent. Math. 89 (1987) 37-118 MR892187

[18] M Harris, D Soudry, $\mathbf{R}$ Taylor, l-adic representations associated to modular forms over imaginary quadratic fields I: Lifting to $\mathrm{GSp}_{4}(\mathbb{Q})$, Invent. Math. 112 (1993) 377411 MR1213108

[19] D Heard, Orb: a program for computing hyperbolic structures on orbifolds Available at http://www.ms.unimelb.edu.au/ snap/orb.html

[20] H M Hilden, M T Lozano, J M Montesinos-Amilibia, On the arithmetic 2-bridge knots and link orbifolds and a new knot invariant, J. Knot Theory Ramifications 4 (1995) 81-114 MR1321291

[21] J Hoste, PD Shanahan, Commensurability classes of twist knots, J. Knot Theory Ramifications 14 (2005) 91-100 MR2124555

[22] H Jacquet, R P Langlands, Automorphic forms on GL(2), Springer, Berlin (1970) MR0401654

[23] R C Kirby, Problems in low-dimensional topology, from: "Geometric topology (Athens, GA, 1993)", AMS/IP Stud. Adv. Math. 2, Amer. Math. Soc., Providence, RI (1997) 35-473 MR1470751 
[24] J-P Labesse, J Schwermer, On liftings and cusp cohomology of arithmetic groups, Invent. Math. 83 (1986) 383-401 MR818358

[25] A Lubotzky, Group presentation, p-adic analytic groups and lattices in $\mathrm{SL}_{2}(\mathbb{C})$, Ann. of Math. (2) 118 (1983) 115-130 MR707163

[26] A Lubotzky, Eigenvalues of the Laplacian, the first Betti number and the congruence subgroup problem, Ann. of Math. (2) 144 (1996) 441-452 MR1418904

[27] A Lubotzky, Free quotients and the first Betti number of some hyperbolic manifolds, Transform. Groups 1 (1996) 71-82 MR1390750

[28] C Maclachlan, A W Reid, The arithmetic of hyperbolic 3-manifolds, Graduate Texts in Mathematics 219, Springer, New York (2003) MR1937957

[29] J Martinet, Petits discriminants des corps de nombres, from: "Number theory days, 1980 (Exeter, 1980)", London Math. Soc. Lecture Note Ser. 56, Cambridge Univ. Press, Cambridge (1982) 151-193 MR697261

[30] J J Millson, On the first Betti number of a constant negatively curved manifold, Ann. of Math. (2) 104 (1976) 235-247 MR0422501

[31] M F Newman, EA O'Brien, Application of computers to questions like those of Burnside II, Internat. J. Algebra Comput. 6 (1996) 593-605 MR1419133

[32] G Perelman, The entropy formula for the Ricci flow and its geometric applications arXiv:math.DG/0211159

[33] G Perelman, Ricci flow with surgery on three-manifolds arXiv:math.DG/0303109

[34] C S Rajan, On the non-vanishing of the first Betti number of hyperbolic three manifolds, Math. Ann. 330 (2004) 323-329 MR2089429

[35] M Rapoport, T Zink, Über die lokale Zetafunktion von Shimuravarietäten. Monodromiefiltration und verschwindende Zyklen in ungleicher Charakteristik, Invent. Math. 68 (1982) 21-101 MR666636

[36] J D Rogawski, Automorphic representations of unitary groups in three variables, Annals of Mathematics Studies 123, Princeton University Press, Princeton, NJ (1990) MR1081540

[37] R Schoof, Abelian varieties over cyclotomic fields with good reduction everywhere, Math. Ann. 325 (2003) 413-448 MR1968602

[38] H P F Swinnerton-Dyer, On l-adic representations and congruences for coefficients of modular forms II, from: "Modular functions of one variable, V (Proc. Second Internat. Conf., Univ. Bonn, Bonn, 1976)”, Springer, Berlin (1977) 63-90. Lecture Notes in Math., Vol. 601 MR0498392

[39] J Tate, The non-existence of certain Galois extensions of $\mathbb{Q}$ unramified outside 2, from: "Arithmetic geometry (Tempe, AZ, 1993)", Contemp. Math. 174, Amer. Math. Soc., Providence, RI (1994) 153-156 MR1299740 
[40] R Taylor, $l$-adic representations associated to modular forms over imaginary quadratic fields II, Invent. Math. 116 (1994) 619-643 MR1253207

[41] R Taylor, On Galois representations associated to Hilbert modular forms II, from: "Elliptic curves, modular forms \& Fermatś last theorem (Hong Kong 1993)", Ser. Number Theory I, International Press (1995) 185-191

[42] R Taylor, A Wiles, Ring-theoretic properties of certain Hecke algebras, Ann. of Math. (2) 141 (1995) 553-572 MR1333036

[43] R Taylor, T Yoshida, Compatibility of local and global Langlands correspondences, preprint (2004)

[44] The GAP Group, GAP - Groups, Algorithms and Programming, version 4.2 (2000) Available at http://www-gap.dcs.st-and.ac.uk/ gap/

[45] M-F Vignéras, Arithmétique des algèbres de quaternions, Lecture Notes in Mathematics 800, Springer, Berlin (1980) MR580949

[46] F Waldhausen, The word problem in fundamental groups of sufficiently large irreducible 3-manifolds, Ann. of Math. (2) 88 (1968) 272-280 MR0240822

[47] J R Weeks, SnapPea: a computer program for investigating hyperbolic 3-manifolds Available at http://www.geometrygames.org/

[48] A Weil, On discrete subgroups of Lie groups, Ann. of Math. (2) 72 (1960) 369-384 MR0137792

[49] A Wiles, Modular elliptic curves and Fermat's last theorem, Ann. of Math. (2) 141 (1995) 443-551 MR1333035

Department of Mathematics, Harvard University

One Oxford Street, Cambridge MA 02138, USA

Mathematics 253-37, Caltech

Pasadena CA 91125, USA

fcale@math.harvard.edu, dunfield@caltech.edu

http://www.math.harvard.edu/ fcale/,

http://www.its.caltech.edu/ dunfield/

Proposed: Walter Neumann

Seconded: David Gabai, Tomasz Mrowka
Received: 18 August 2005

Revised: 28 February 2006 